\title{
Uma Abordagem para Jogos Bayesianos Fuzzy com base na Probabilidade Fuzzy de Buckley ${ }^{1}$
}

\author{
Tiago da Cruz Asmus ${ }^{2}$ \\ Graçaliz Pereira Dimuro ${ }^{23}$
}

\begin{abstract}
Resumo: Este artigo apresenta uma abordagem para jogos Bayesianos Fuzzy, com base na noção de probabilidades fuzzy para estimar tipos desconhecidos de jogadores envolvidos em situações estratégicas. Para tal, considera-se a noção de probabilidade fuzzy introduzida por Buckley e Eslami, que utilizam números fuzzy para representar probabilidades incertas e imprecisas, sem, contudo empregar a teoria da probabilidade fuzzy, mas considerando uma restrição aritmética ao intervalo $[0 ; 1]$. $\mathrm{O}$ artigo formaliza essa noção de probabilidade fuzzy, definindo jogos Bayesianos fuzzy para 2 jogadores com $n$ tipos possíveis. Discute-se também, através de exemplos, a dependência da solução do modelo da relação de ordem adotada no espaço de números fuzzy. Propõe-se a utilização de relação de ordem de Asmus-Dimuro para determinação da solução de equilíbrio de Nash.
\end{abstract}

\begin{abstract}
This paper presents an approach for fuzzy Bayesian games, based on the notion of fuzzy probabilities to estimate unknown types of agents in strategic situations. For that, we consider the notion of fuzzy probability introduced by Buckley and Eslami, who use fuzzy numbers to represent uncertain and imprecise probabilities, without, however, applying the fuzzy probability theory, but considering an arithmetic restriction to the interval $[0 ; 1]$. The paper formalizes this notion of fuzzy probability, defining fuzzy Bayesian games of 2 agents with $n$ possible types. We also discuss, by analyzing some examples, the dependence of the model solution on the order relation adopted in the fuzzy number space. Then, we suggest the use of the total order relation of Asmus-Dimuro in order to find the Nash equilibrium solution.
\end{abstract}

\section{Introdução}

A teoria dos jogos constitui uma base importante para simular diversas situações onde vários agentes (denominados de jogadores) interagem de forma estratégica para tomada de

\footnotetext{
${ }^{1}$ Este artigo é versão estendida do trabalho apresentado no WEIT 2011 e classificado como um dos três melhores artigo do evento.

${ }^{2}$ Programa de Pós-Graduação em Modelagem Computacional, Universidade Federal do Rio Grande - FURG, Av. Itália km 08, Campus Carreiros, 96200-970 - Rio Grande, RS, Brasil, e-mail: \{tiagoasmus s gmai l . com\} ${ }^{3}$ Programa de Pós-Graduação em Modelagem Computacional, Programa de Pós-Graduação em Computação, Centro de Ciências Computacionais, Universidade Federal do Rio Grande - FURG, Av. Itália km 08, Campus Carreiros, 96200-970 - Rio Grande, RS, Brasil, e-mail: \{gracalizegmail.com\}
} 
decisão. Têm sido largamente utilizada em várias áreas, tais como ciências sociais, econômicas, biológicas e computacionais. São várias as aplicações em competição, votação, leilões, corrida de desenvolvimento e pesquisa, comportamento de cartéis, comércio eletrônico, inteligência artificial, sistemas multiagentes, entre outros. [34, 35, 37, 43, 50, 52, 54, 56]

Os jogos podem ser classificados em duas grandes classes: jogos cooperativos e não cooperativos. No primeira abordagem, os jogadores focam a formação de coalisões, enquanto que, na última, o foco é na escolha de estratégias. Este trabalho situa-se na classe de jogos não cooperativos.

Em algumas situações, os jogadores podem desconhecer algumas características-chaves dos outros jogadores envolvidos na interação estratégica, ou até mesmo de si mesmos (por exemplo, o perfil de estratégias dos diferentes jogadores, suas preferências e/ou as preferências dos outros jogadores), configurando um jogo de informação incompleta. [32]

Para contornar esse problema, Harsanyi [32] $]^{1}$ sugeriu que as informações privadas sobre os jogadores constituíssem o tipo do jogador e que fossem estimadas probabilidades para cada tipo desconhecido, introduzindo o denominado Jogo Bayesiano. ${ }^{2}$

Considerando que, neste caso, o jogo passa a produzir recompensas probabilísticas para cada jogador, um jogo com informação incompleta pode ser tratado como um jogo com informação imperfeita. Com base nesta suposição, vários autores consideraram jogos com recompensas randômicas, entre eles, Blau [8], Cassidy et al. [19], Gao et al. [29], Charnes et al. [21], Berg [7] e Roberts [48]. ${ }^{3}$

Por outro lado, existem casos em que é muito difícil caracterizar a informação particular de cada agente (por exemplo, a habilidade, o nível de esforço, ou a personalidade de um agente) [54]. Neste caso, as recompensas são dadas por valores aproximados, vagos, nebulosos (e não probabilísticos), e o conceito de estratégia de equilíbrio de Nash Bayesiano não pode ser aplicado no contexto do jogo. Tem-se então na teoria dos conjuntos fuzzy [60, 61] uma excelente base para estudar esse tipo de jogo onde as recompensas são representadas por números fuzzy.

Dang e Hong [23] identificaram duas linhas principais de pesquisa sobre jogos fuzzy: os jogos matriciais fuzzy e os jogos não cooperativos fuzzy, discutindo os diversos trabalhos desenvolvidos nestas duas direções, onde a maioria está focada em jogos de soma zero.

Butnariu $[16,17]$ foi o primeiro a introduzir a teoria dos conjuntos fuzzy em jogos não cooperativos para produzir esse novo tipo de jogo fuzzy.

\footnotetext{
${ }^{1}$ John Charles Harsanyi (1920-2000), economista húngaro. Recebeu o Prêmio de Ciências Econômicas em Memória de Alfred Nobel em 1994.

${ }^{2}$ Outros autores contribuíram para o desenvolvimento da teoria de jogos Bayesianos, entre eles, Myerson [42] e Aumann [4].

${ }^{3}$ Para uma visão geral sobre jogos com informação incompleta, veja [57].
} 

ley ${ }^{1}$

Campos [18] foi o primeiro a propor o jogo matricial fuzzy com recompensas fuzzy, baseado no estabelecimento de uma programação linear fuzzy para cada jogador, mas não definiu explicitamente conceitos de uma estratégia de equilíbrio. Seguindo por um caminho semelhante, mas considerando apenas jogos bimatriciais com recompensas fuzzy, Maeda [38, 39] definiu dois tipos de conceitos de estratégias de equilíbrio e investigou suas relações. Já Nishizaki e Sakawa [46] discutiram o jogo matricial com multiobjetivos e recompensas fuzzy.

Garagic e Cruz [30] consideraram jogos estáticos com $n$ jogadores e o conceito de solução neste contexto. Vijay et al. [51] investigaram a solução de jogos matriciais de soma zero com dois jogadores, onde os objetivos e as recompensas são números fuzzy. Xu et al. [59] definiram três estratégias de equilíbrio para jogos de soma zero com dois jogadores com recompensas fuzzy, sendo essas recompensas caracterizadas como variáveis fuzzy em um espaço de credibilidade. Larbani [34] apresentou uma revisão sobre diversas abordagens para a resolução de jogos fuzzy não cooperativos na forma normal, apresentando algumas aplicações e introduzindo uma classe de jogos não cooperativos envolvendo parâmetros fuzzy. Kacher e Larbani [33] estudaram a existência de uma solução de equilíbrio para um jogo não cooperativo com objetivos fuzzy envolvendo parâmetros fuzzy.

Amaral e Gomide [1, 2, 3] analisaram estratégias de equilíbrio e desenvolveram métodos computacionais para a resolução de jogos fuzzy, tanto cooperativos como não-cooperativos, com base em computação evolutiva, em $\alpha$-níveis e algoritmos de decomposição. Mais recentemente, Dang e Hong [23] consideraram o jogo de Cournot com demanda e custos fuzzy utilizando números fuzzy triangulares.

Gao [28] apresentou um apanhado geral sobre jogos de credibilidade onde a informação incompleta é interpretada através de variáveis fuzzy e tratada à luz da teoria da credibilidade.

Entretanto, em diversos problemas e aplicações reais, observa-se que a informação pode ser incompleta em dois sentidos: a chance de um jogador ser de um tipo ou outro é probabilística, mas, além disso, o conhecimento sobre cada tipo é fuzzy. Wang et al. [54] discutiram esse tipo de problema, apresentando como exemplos as competições de futebol (a chance de dois times se encontrarem em uma partida é randômica e o comportamento de cada time é fuzzy) e o modelo de competição de Cournot com informação assimétrica (a eficiência de duas empresas é usualmente caracterizada como variáveis fuzzy randômicas com valores fuzzy, por exemplo, baixa ou alta).

Assim, Xu et al. [58] introduziram um jogo matricial de soma zero com dois jogadores onde as recompensas são tratadas como variáveis randômicas fuzzy. Liu e Kao [36] desenvolveram um método de solução para esse tipo de jogo onde as recompensas são parcialmente conhecidas, e assim, representadas por números fuzzy. Wang et al. [54] introduziram um jogo especial com informação incompleta, onde as recompensas são aleatórias e fuzzy, e as regras 
de decisão para os jogadores são baseadas na teoria de credibilidade. Buckley e Jowers [15] empregaram métodos de Monte Carlo Fuzzy para obter estratégias mistas fuzzy ótimas para jogos de soma zero de dois jogadores.

Neste artigo, introduz-se um modelo de jogo de informação incompleta não cooperativo com 2 jogadores e $l \times m$ combinações de tipos de jogadores, onde as probabilidades incertas atribuídas aos tipos dos jogadores, e, por conseguinte, as recompensas, são números fuzzy triangulares simétricos ${ }^{4}$, configurando assim um jogo Bayesiano fuzzy.

Nesta abordagem proposta, a nebulosidade das probabilidades é considerada como a dificuldade de um especialista em estimar a distribuição de probabilidade do conjunto de tipos de cada jogador. Para modelar essa incerteza, e auxiliar o processo de tomada de decisão em uma situação estratégica, utilizam-se probabilidades fuzzy, obedecendo a uma restrição aritmética proposta por Buckley e Eslami [13, 14] para o cálculo de recompensas probabilísticas. Buckley e Esalami [14] utilizaram números fuzzy para representar probabilidades incertas e imprecisas, sem, contudo empregar a teoria da probabilidade fuzzy no modelo. Utilizando uma álgebra fuzzy restrita ao intervalo [0;1], apresentaram um método para calcular probabilidades fuzzy, valores esperados, variância, média, e estudaram diversas propriedades de probabilidades incertas. Esta abordagem permitiu uma formulação bem mais simplificada para o modelo de jogo Bayesiano fuzzy introduzido neste artigo do que as baseadas na teoria da credibilidade.

Observa-se que não é realizado, neste artigo, um estudo formal geral sobre as condições de existência ou não de soluções de equilíbrio de Nash Bayesiano Fuzzy para o modelo proposto. Entretanto, exemplos com cálculo de soluções de equilíbrio são apresentados, e analisam-se, através desses exemplos, a dependência da solução do modelo da relação de ordem adotada no espaço de números fuzzy.

Na literatura, existem vários métodos de ordenação de números fuzzy, tais como os apresentados em [10, 11, 12, 13, 14, 20, 31, 44, 53, 55]. Em geral, esses métodos são definidos através de relações de ordem parcial, mas não total. Assim, com a utilização desses métodos, nem sempre é possível obter uma solução de equilíbrio de Nash Bayesiano Fuzzy, outras vezes tem-se várias soluções. Desta forma, propõe-se a utilização da ordem de AsmusDimuro [26], que é uma relação de ordem total no espaço de números fuzzy triangulares simétricos, comparando os resultados obtidos com a relação de ordem proposta por Buckley e Esalami. [13, 14]

Este artigo está organizado como descrito a seguir. Na Seção 2, apresentam-se conceitos básicos sobre conjuntos e números fuzzy, necessários para a apresentação do modelo. Os métodos de ordenação de números fuzzy são discutidos na Seção 3.

\footnotetext{
${ }^{4}$ Embora números fuzzy triangulares simétricos não sejam fechados para a divisão, optou-se por essa representação em função de sua simplicidade, uma vez que na abordagem proposta para aplicação em jogos Bayesianos fuzzy não se faz uso de probabilidades condicionais.
} 
Uma Abordagem para Jogos Bayesianos Fuzzy com base na Probabilidade Fuzzy de Buckley ${ }^{1}$

A abordagem adotada para probabilidades fuzzy é apresentada na Seção 4, onde também introduziu-se uma formalização mais geral para a apresentação da restrição aritmética de Buckley-Esalami, em termos de vetores estocásticos.

A Seção 5 apresenta conceitos básicos sobre a Teoria dos Jogos, em particular, Jogos Bayesianos. O modelo de Jogo Bayesiano Fuzzy proposto, onde o cálculo de probabilidade fuzzy obedece à restrição aritmética de Buckley-Esalami, é introduzido na Seção 6. A Seção 7 apresenta exemplos, onde busca-se a solução de equilíbrio e analisa-se a dependência desta solução da relação de ordem adotada.

A Seção 8 é a Conclusão.

\section{Conjuntos Fuzzy}

Nesta Seção, apresentam-se conceitos básicos da Teoria dos Conjuntos Fuzzy ${ }^{5}$, que foi introduzida por Zadeh $[60,61]$. A noção principal desta teoria é a de função de pertinência, que é uma extensão da função característica da teoria clássica de conjuntos. Observe que, se $F$ é um subconjunto de $U$, então sua função característica $\chi_{F}: U \rightarrow\{0,1\}$ é dada por:

$$
\chi_{F}(x)= \begin{cases}1, & \text { se } x \in F \\ 0, & \text { se } x \notin F,\end{cases}
$$

significando que $x \in F$ quando $\chi_{F}(x)=1$ e que $x \notin F$ quando $\chi_{F}(x)=0$.

Quando se admite que um elemento $x \in U$ pode pertencer gradualmente a $F$, então $F$ é um subconjunto fuzzy de $U$ e pode-se definir uma função $\varphi_{F}: U \rightarrow[0 ; 1]$, chamada de função de pertinência do conjunto fuzzy $F$. O valor de $\varphi_{F}(x)$ indica o grau com que $x$ pertence ao conjunto $F$, onde $\varphi_{F}(x)=0$ e $\varphi_{F}(x)=1$ representam, respectivamente, a não pertinência completa e a pertinência completa de $x$ ao conjunto fuzzy $F$. Assim, um conjunto clássico, ou crisp, passa a ser um caso particular de um conjunto fuzzy, onde sua função de pertinência $\varphi_{F}$ é a própria função característica $\chi_{F}$.

Um subconjunto fuzzy $F$ de $U$ pode ser representado por um conjunto de pares ordenados da forma $\left(x, \varphi_{F}(x)\right)$, com $x \in U$, atribuindo assim um grau de pertinência em $F$ para cada elemento do domínio de $U$ :

$$
F=\left\{\left(x, \varphi_{F}(x)\right) \mid x \in U\right\} .
$$

Denomina-se suporte de $F$ o conjunto

$$
\operatorname{supp}_{F}=\left\{x \in U \mid \varphi_{F}(x)>0\right\},
$$

${ }^{5}$ Para maiores detalhes sobre a teoria dos conjuntos fuzzy, consulte [5, 9, 22, 45, 49]. 
ou seja, o conjunto de todos os elementos de $U$ que pertençam, mesmo que parcialmente, ao conjunto $F$. Denominam-se $\alpha$-níveis de $F$ os subconjuntos clássicos de $U$ definidos por

$$
F[\alpha]=\left\{x \in U \mid \varphi_{F}(x) \geq \alpha\right\},
$$

para $0<\alpha \leq 1$, e, para $\alpha=0$, como o fecho do suporte de $F$, isto é,

$$
F[0]=\widehat{\operatorname{suppF}_{F}},
$$

onde $\operatorname{supp}_{F}$ é o suporte de $F$ definido na Eq. (2). Um conjunto fuzzy é completamente definido através de seus $\alpha$-níveis. [5]

Um conjunto fuzzy $\bar{F}$ é chamado de número fuzzy quando $\varphi_{\bar{F}}$ está definida no conjunto dos números reais $\mathrm{R}$, e as seguintes condições são satisfeitas [5]:

a) todos os $\alpha$-níveis de $\bar{F}$ são não vazios, com $0 \leq \alpha \leq 1$;

b) todos os $\alpha$-níveis de $\bar{F}$ são intervalos fechados de $\mathrm{R}$;

c) o suporte de $\bar{F}$ é limitado. $\operatorname{como}^{6}$ :

Um número fuzzy $\bar{F}$ é dito triangular se possuir uma função de pertinência definida

$$
\varphi_{\bar{F}}(x)= \begin{cases}0, & \text { se } x \leq a \\ \frac{x-a}{u-a}, & \text { se } a<x \leq u \\ \frac{x-b}{u-b}, & \text { se } u<x \leq b \\ 0, & \text { se } x \geq b,\end{cases}
$$

para $a, u, b \in \mathrm{R}$. Assim, o número fuzzy triangular $\bar{F}$ é determinado pelos números reais $a$, $u$ e $b$, e é denotado pela terna ordenada $(a / u / b)$, onde $u$ é chamado de core desse número fuzzy, pois é o único valor que pertence completamente ao conjunto $\bar{F}$, ou seja, $\varphi_{\bar{F}}(u)=1$.

O gráfico da função de pertinência de um número fuzzy triangular pode ser visto na Figura 1. O suporte de $\vec{F}$ é um intervalo aberto dados por $\operatorname{supp}_{\bar{F}}=(a ; b)$, e sua representação através de seus $\alpha$-níveis é

$$
\bar{F}[\alpha]=[(u-a) \alpha+a,(u-b) \alpha+b] .
$$

${ }^{6}$ Veja [5] para outros tipos de funções de pertinência 
Uma Abordagem para Jogos Bayesianos Fuzzy com base na Probabilidade Fuzzy de Buckley ${ }^{1}$

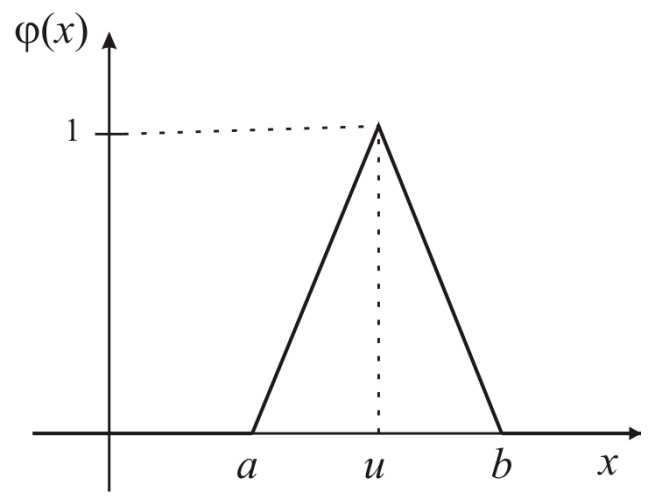

Figura 1. Função de pertinência de um número fuzzy triangular

Observe que os $\alpha$-níveis de um número fuzzy são intervalos fechados. Logo, para fazer operações com números fuzzy, pode-se representá-los por meio de seus $\alpha$-níveis e operá-los com aritmética intervalar [41].

De particular interesse para este trabalho são os números fuzzy triangulares simétricos, quando $b-u=u-a$, pois eles são utilizados para modelar a expressão "em torno de".

\section{Ordenação de Números Fuzzy}

Dados dois números fuzzy $\bar{F}_{1}$ e $\bar{F}_{2}$, existem diversos métodos de ordenação para determinar se $\bar{F}_{1} \leq \bar{F}_{2}$ (por exemplo: [10,11,12,13,14, 20,31, 44, 53, 55]). A escolha de qual método de ordenação a utilizar depende das formas de $\bar{F}_{1}$ e $\bar{F}_{2}$ e do problema que está sendo modelado.

Para este trabalho, dados números fuzzy $\bar{F}_{1}$ e $\bar{F}_{2}$ e seus respectivos $\alpha$-níveis

$$
\bar{F}_{1}[\alpha]=\left[a_{1}(\alpha), a_{2}(\alpha)\right] \text { e } \bar{F}_{2}[\alpha]=\left[b_{1}(\alpha), b_{2}(\alpha)\right],
$$

consideram-se as seguintes relações, as duas primeiras propostas por Buckley $[13,14]$ (para aplicações envolvendo probabilidades fuzzy):

Relação de ordem (1) [13, Cap. 14, pag. 145]:

$$
\bar{F}_{1} \leq \bar{F}_{2} \Leftrightarrow \forall \alpha: \bar{F}_{1}[\alpha] \leq \bar{F}_{2}[\alpha]
$$

onde

$$
\forall \alpha \in[0 ; 1]: \bar{F}_{1}[\alpha] \leq \bar{F}_{2}[\alpha] \Leftrightarrow a_{1}(\alpha) \leq b_{1}(\alpha) \wedge a_{2}(\alpha) \leq b_{2}(\alpha) .
$$


Observe que a relação $\leq$ definida na Eq. (9) constitui a relação de ordem parcial de Kulisch-Miranker da Matemática Intervalar, possuindo as seguintes propriedades para números fuzzy $\bar{F}_{1}, \bar{F}_{2}$ e $\bar{F}_{3}:[40,41]$

(a) Reflexão: $\bar{F}_{1} \leq \bar{F}_{1}$;

(b) Anti-simetria: $\bar{F}_{1} \leq \bar{F}_{2} \wedge \bar{F}_{2} \leq \bar{F}_{1} \Rightarrow \bar{F}_{1}=\bar{F}_{2}$;

(c) Transitividade: $\bar{F}_{1} \leq \bar{F}_{2} \wedge \bar{F}_{2} \leq \bar{F}_{3} \Rightarrow \bar{F}_{1} \leq \bar{F}_{3}$.

Assim, $\leq$ definida na Eq. (8) representa uma relação de ordem. É imediato que é uma relação parcial $[40,41]$.

\section{Relação de ordem (2) [14]:}

$$
\bar{F}_{1} \leq \bar{F}_{2} \Leftrightarrow \forall \alpha \in[0 ; 1]: \bar{F}_{1}[\alpha] \subseteq \bar{F}_{2}[\alpha] .
$$

A relação $\leq$ dada na Eq. (10) é definida com base na relação de inclusão de intervalos, também estudada na Matemática Intervalar, possuindo as propriedades (a), (b) e (c) citadas anteriormente. Logo, também constitui uma relação de ordem parcial, mas não total. [40, 41]

\section{Relação de ordem de Asmus-Dimuro [26]:}

A relação de ordem introduzida por Asmus e Dimuro [26] para números fuzzy triangulares simétricos estende a relação de ordem (1) e reinterpreta a relação de ordem (2).

Considere os números fuzzy triangulares simétricos

$$
\bar{F}_{1}=\left(a_{1} / u_{1} / b_{1}\right) \text { e } \bar{F}_{2}=\left(a_{2} / u_{2} / b_{2}\right) .
$$

Existem diversas formas de representar seus respectivos $\alpha$-níveis, como

$$
\begin{aligned}
& \bar{F}_{1}[\alpha]=\left[\left(u_{1}-a_{1}\right) \alpha+a_{1},\left(u_{1}-b_{1}\right) \alpha+b_{1}\right] \\
& \bar{F}_{2}[\alpha]=\left[\left(u_{2}-a_{2}\right) \alpha+a_{2},\left(u_{2}-b_{2}\right) \alpha+b_{2}\right] .
\end{aligned}
$$

As expressões (11) e (12) podem ser reescritas de forma a omitir os cores dos números fuzzy, que são trivialmente obtidos através de uma média aritmética simples, pois os números são triangulares simétricos. Tem-se, então, a seguinte representação:

$$
\begin{aligned}
& \bar{F}_{1}[\alpha]=\left[\frac{b_{1} \alpha+(2-\alpha) a_{1}}{2}, \frac{a_{1} \alpha+(2-\alpha) b_{1}}{2}\right] \\
& \bar{F}_{2}[\alpha]=\left[\frac{b_{2} \alpha+(2-\alpha) a_{2}}{2}, \frac{a_{2} \alpha+(2-\alpha) b_{2}}{2}\right] .
\end{aligned}
$$


Uma Abordagem para Jogos Bayesianos Fuzzy com base na Probabilidade Fuzzy de Buckley ${ }^{1}$

Outra forma interessante de se representar alfa-níveis de números fuzzy triangulares simétricos é através do conceito de dispersão, ou seja, a distância do core até os extremos do número em questão. Essa representação se dá da seguinte forma:

$$
\begin{aligned}
& \bar{F}_{1}[\alpha]=\left[h_{1} \alpha+\left(u_{1}-h_{1}\right),-h_{1} \alpha+\left(u_{1}+h_{1}\right)\right] \\
& \bar{F}_{2}[\alpha]=\left[h_{2} \alpha+\left(u_{2}-h_{2}\right),-h_{2} \alpha+\left(u_{2}+h_{2}\right)\right] .
\end{aligned}
$$

onde $h_{1}=u_{1}-a_{1}$ e $h_{2}=u_{2}-a_{2}$ são as dispersões de $\bar{F}_{1}$ e $\bar{F}_{2}$, respectivamente.

Para a elaboração do método de ordenação pretendido será utilizada a representação contida nas expressões (11) e (12), pois ela facilita a visualização de alguns casos onde o resultado da ordenação não é trivial.

Primeiramente, a relação de igualdade é definida como:

$$
\bar{F}_{1}=\bar{F}_{2} \Leftrightarrow a_{1}=a_{2} \wedge b_{1}=b_{2} .
$$

Para definir a relação $<$, utiliza-se um grau de imprecisão $\rho \in(0 ; 1]$ que determina o quanto os valores dos cores dos números comparados vão ser determinantes para ordenar números fuzzy muito próximos. Tomando valores de $\rho$ próximos de zero, dá-se mais importância à precisão da informação contida no número fuzzy do que na quantidade numérica que ele representa. Analisando esse caso graficamente, números fuzzy "estreitos" tendem a ser maiores do que números fuzzy "largos", pois os primeiros carregam melhor informação do que os últimos. $[6,24,25]$

Assim, a relação $<$ é definida por quatro condições, bastando que uma delas se verifique para a relação ser verdadeira. Essas condições abrangem todas as possibilidades de posicionamento entre dois números fuzzy triangulares simétricos, levando em consideração ainda o grau de imprecisão $\rho$ determinado de acordo com o problema modelado [26]. Então, define-se:

$$
\begin{aligned}
& \bar{F}_{1}<\bar{F}_{2} \Leftrightarrow \\
& \left(a_{1}<a_{2}\right) \wedge\left(b_{1} \leq b_{2}\right) \\
& \vee\left(a_{1}<a_{2}\right) \wedge\left(b_{2}<b_{1}\right) \wedge\left(u_{1} \leq u_{2}\right) \\
& \vee\left(a_{1}<a_{2}\right) \wedge\left(b_{2}<b_{1}\right) \wedge\left(u_{2}<u_{1}\right) \wedge \\
& {\left[\forall \alpha:(0 \leq \alpha \leq \rho) \wedge\left(u_{1}-a_{1}\right) \alpha+a_{1} \leq\left(u_{2}-a_{2}\right) \alpha+a_{2}\right]} \\
& \vee\left(a_{2} \leq a_{1}\right) \wedge\left(\left(b_{1}<b_{2}\right) \wedge\left(u_{1}<u_{2}\right) \wedge\right. \\
& {\left[\exists \alpha:(0 \leq \alpha \leq \rho) \wedge\left(u_{1}-a_{1}\right) \alpha+a_{1}<\left(u_{2}-a_{2}\right) \alpha+a_{2}\right] .}
\end{aligned}
$$


Com base nas equações (17) e (18), define-de para quaisquer números fuzzy triangulares simétricos $\bar{F}_{1}$ e $\bar{F}_{2}$ :

$$
\bar{F}_{1} \leq \bar{F}_{2} \Leftrightarrow \bar{F}_{1}=\bar{F}_{2} \vee \bar{F}_{1}<\bar{F}_{2}
$$

Tem-se que esta relação satisfaz as propriedades de reflexão, anti-simetria e transitividade, e, portanto, é uma relação de ordem. Além disso, é válido que

$$
\forall \bar{F}_{1}, \bar{F}_{2}: \bar{F}_{1}=\bar{F}_{2} \vee \bar{F}_{1}<\bar{F}_{2} \vee \bar{F}_{2}<\bar{F}_{1},
$$

e, portanto, constitui uma relação de ordem total. [26]

\section{A Abordagem de Buckley para Probabilidade Fuzzy}

Nesta seção, apresenta-se uma formalização geral, em termos de vetores estocásticos, para a abordagem proposta por Buckley para cálculo de probabilidades fuzzy, a qual não trabalha com a Teoria de Probabilidade Fuzzy. [13, 14]

Seja $X=\left\{x_{1}, \ldots, x_{n}\right\}$ um conjunto finito e $P: \wp(X) \rightarrow[0 ; 1]$ uma função de probabilidade definida para todos os subconjuntos de $X$, tal que com $P\left(\left\{x_{i}\right\}\right)=\phi_{i}$, com $1 \leq i \leq n, 0 \leq \phi_{i} \leq 1$ e $\sum_{i=1}^{n} \phi_{i}=1$. Logo, $(X, P)$ caracteriza uma distribuição de probabilidades finita e discreta, representada pelo conjunto $\Phi=\left\{\phi_{1}, \ldots, \phi_{n}\right\}$.

Observa-se que os elementos de $\Phi$ muitas vezes são valores obtidos pela opinião de especialistas, e nem sempre representam valores precisos e de consenso. Para modelar essa incerteza, podem-se utilizar números fuzzy, e, assim, todos os $\phi_{i} \in \Phi$ são substituídos por números fuzzy $\bar{\phi}_{i}$, sendo esse novo conjunto denotado por $\bar{\Phi}$ (mesmo que apenas alguns dos elementos de $\bar{\Phi}$ sejam imprecisos). Para os valores conhecidos e exatos, ainda se utilizará a notação fuzzy, mesmo que eles representem números crisp.

Assim, a função de probabilidade fuzzy $\bar{P}$ será a representação da opinião incerta de um especialista sobre o assunto estudado. Os elementos de $\bar{\Phi}$ serão números fuzzy triangulares simétricos da forma

$$
\bar{P}\left(\left\{x_{i}\right\}\right)=\bar{\phi}_{i}=\left(a_{i} / u_{i} / b_{i}\right),
$$

para $1 \leq i \leq n$, onde $a_{i}$ representa a estimativa de ocorrência pessimista de $x_{i}, b_{i}$ a otimista e $u_{i}$ a "mais aceitável".

Quando vários especialistas fornecem esses valores, então $a_{i}$ e $b_{i}$ serão obtidos através do cálculo da variância de todas as estimativas pessimistas e otimistas, respectivamente, sobre a ocorrência de $x_{i}$, e $u_{i}$ será o resultado da média aritmética de todas as estimativas "mais aceitáveis" sobre o evento em questão. [13] 
Uma Abordagem para Jogos Bayesianos Fuzzy com base na Probabilidade Fuzzy de Buckley ${ }^{1}$

Para que $(X, \bar{\Phi})$ represente de fato uma distribuição finita e discreta de probabilidades, é necessário que se faça uma restrição aritmética, pois a soma intervalar dos $\alpha$-níveis de todos os $\bar{\phi}_{i}$ só resultará em 1 no caso de todos os intervalos serem degenerados, o que dificilmente acontece. [13, 14]

Então, para todo $0 \leq \alpha \leq 1$, escolhe-se de cada $\alpha$-nível $\bar{\phi}_{i}[\alpha]$ um $e_{i}$ tal que $\sum_{i=1}^{n} e_{i}=$ 1. Ou seja, ao invés da soma intervalar dos $\alpha$-níveis resultar em 1, basta que se obtenha apenas um valor crisp $e_{i}$ para cada intervalo $\bar{\phi}_{i}[\alpha]$ que somados resultem em 1 .

Para definir formalmente essa restrição aritmética, considera-se o conjunto de vetores estocásticos:

$$
E=\left\{\left(e_{1}, \ldots, e_{n}\right) \in[0 ; 1]^{n} \mid \sum_{i=1}^{n} e_{i}=1\right\}
$$

e o conjunto $\mathcal{A}_{\bar{\Phi}}^{\alpha}$ formado pelo produto cartesiano dos $\alpha$-níveis dos elementos de $\bar{\Phi}$ :

$$
\mathcal{A}_{\bar{\Phi}}^{\alpha}=\bar{\phi}_{1}[\alpha] \times \ldots \times \bar{\phi}_{n}[\alpha] .
$$

Obtém-se então o conjunto

$$
\mathcal{S}_{\bar{\Phi}}^{\alpha}=\mathcal{A}_{\bar{\Phi}}^{\alpha} \cap E,
$$

onde $E$ é dado na Eq. (21) e $\mathcal{A}_{\bar{\Phi}}^{\alpha}$ na Eq. (22). O conjunto $\mathcal{S}_{\bar{\Phi}}^{\alpha}$ será o domínio da função que caracterizará a restrição aritmética pretendida.

Considere um subconjunto $A \subseteq X$ e seja

$$
I_{A}^{X}=\left\{i \in\{1, \ldots, n\} \mid x_{i} \in A\right\}
$$

o conjunto dos índices dos elementos de $A$ relativos aos índices do conjunto $X=\left\{x_{1}, \ldots, x_{n}\right\}$. Quando o conjunto de índices de $X$ pode ser entendido pelo contexto, denota-se o conjunto de índices de $A \subseteq X$ simplesmente por $I_{A}$. Define-se então a função:

$$
\begin{aligned}
f_{\bar{\Phi}, \alpha}^{A}: \mathcal{S}_{\bar{\Phi}}^{\alpha} & \rightarrow[0 ; 1] \\
\left(e_{1}, \ldots, e_{n}\right) & \mapsto \sum_{i \in I_{A}} e_{i},
\end{aligned}
$$

onde $0 \leq \alpha \leq 1$ e $\mathcal{S}_{\bar{\Phi}}^{\alpha}$ é dado na Eq. (23).

A probabilidade fuzzy de um subconjunto $A \subseteq X$ é obtida, através de seus $\alpha$-níveis, da seguinte forma:

$$
\bar{P}(A)[\alpha]=\left\{f_{\bar{\Phi}, \alpha}^{A}(e) \mid e=\left(e_{1}, \ldots, e_{n}\right) \in \mathcal{S}_{\bar{\Phi}}^{\alpha}\right\},
$$




\section{Buckley $^{1}$}

Uma Abordagem para Jogos Bayesianos Fuzzy com base na Probabilidade Fuzzy de

onde $f_{\bar{\Phi}, \alpha}^{A}: \mathcal{S}_{\bar{\Phi}}^{\alpha} \rightarrow[0 ; 1]$ é a função de restrição aritmética definida na Eq. (25).

Observa-se que primeiro se escolhe uma distribuição de probabilidade discreta completa dos $\alpha$-níveis antes de se calcular uma probabilidade na Eq. (26). Observa-se também que $\bar{P}(A)[\alpha]$ não é a soma dos intervalos $\bar{\phi}_{i}[\alpha]$, com $i \in I_{A}$, usando aritmética intervalar.

Proposition 1. $\bar{P}(A)$ é um número fuzzy.

Prova. É imediato que a função $f_{\bar{\Phi}, \alpha}^{A}$, definida na Eq. (25) para algum $I_{A}$, é contínua, e que seu domínio $\mathcal{S}_{\bar{\Phi}}^{\alpha}$ é formado por intervalos conexos, fechados e limitados, o que implica que a imagem de $f_{\bar{\Phi}, \alpha}^{A}$, dada por $f_{\bar{\Phi}, \alpha}^{A}\left(\mathcal{S}_{\bar{\Phi}}^{\alpha}\right)=\bar{P}(A)[\alpha]$, é um intervalo de números reais, fechado e limitado. Logo, $\bar{P}(A)[\alpha]$ representa os $\alpha$-níveis de um conjunto fuzzy $\bar{P}(A)$. Além disso, $f_{\bar{\Phi}, 1}^{A}\left(\mathcal{S}_{\bar{\Phi}}^{1}\right)=\bar{P}(A)[1] \neq \emptyset$, pois como $\left(\phi_{1}, \ldots, \phi_{n}\right) \in \mathcal{S}_{\bar{\Phi}}^{1}$, então $\sum_{i \in I_{a}} \phi_{i} \in \bar{P}(A)[1]$, o que faz com que $\bar{P}(A)$ seja de fato um número fuzzy.

Mesmo que $\bar{P}(A)$ denote uma probabilidade fuzzy, as seguintes propriedades da probabilidade clássica são mantidas: $[13,14]$
a) $\forall A: 0 \leq \bar{P}(A) \leq 1$;
b) $\bar{P}(\emptyset)=0$;
c) $\bar{P}(X)=1$.

Finalmente, mostra-se como calcular a média fuzzy $\bar{\mu}_{\bar{\Phi}}^{r}$ ponderando as probabilidades fuzzy em $\bar{\Phi}=\left\{\bar{\phi}_{1}, \ldots, \bar{\phi}_{n}\right\}$ por valores reais em $r=\left(r_{1}, \ldots, r_{n}\right) \in \mathrm{R}^{n}$. Para tal, define-se a função $g_{\bar{\Phi}, \alpha}^{r}$, para garantir que a restrição aritmética seja respeitada, como:

$$
\begin{aligned}
g_{\bar{\Phi}, \alpha}^{r}: \mathcal{S}_{\bar{\Phi}}^{\alpha} & \rightarrow \mathrm{R} \\
\left(e_{1}, \ldots, e_{n}\right) & \mapsto \sum_{i=1}^{n} e_{i} \cdot r_{i},
\end{aligned}
$$

onde $0 \leq \alpha \leq 1$ e $\mathcal{S}_{\bar{\Phi}}^{\alpha}$ é dado na Eq. (23).

A média fuzzy para um determinado $r=\left(r_{1}, \ldots, r_{n}\right) \in \mathrm{R}^{n}$ é então definida através de seus $\alpha$-níveis como:

$$
\bar{\mu}_{\bar{\Phi}}^{r}[\alpha]=\left\{g_{\bar{\Phi}, \alpha}^{r}(e) \mid e=\left(e_{1}, \ldots, e_{n}\right) \in \mathcal{S}_{\bar{\Phi}}^{\alpha}\right\}
$$

para $0 \leq \alpha \leq 1$, onde $g_{\bar{\Phi}, \alpha}^{r}: \mathcal{S}_{\bar{\Phi}}^{\alpha} \rightarrow$ R é definida pela Eq. (27).

Proposition 2. $\bar{\mu}_{\bar{\Phi}}^{r}$ é um número fuzzy. 

ley ${ }^{1}$

Prova. É imediato que a função $g_{\bar{\Phi}, \alpha}^{r}$, definida na Eq. (27) para algum $r=\left(r_{1}, \ldots, r_{n}\right) \in \mathrm{R}^{n}$, é contínua, e que seu domínio $\mathcal{S}_{\bar{\Phi}}^{\alpha}$ é formado por intervalos conexos, fechados e limitados, o que implica que a imagem de $g_{\bar{\Phi}, \alpha}^{r}$, dada por $g_{\bar{\Phi}, \alpha}\left(\mathcal{S}_{\bar{\Phi}}^{\alpha}\right)=\bar{\mu}_{\bar{\Phi}}^{r}[\alpha]$, é um intervalo de números reais, fechado e limitado. Logo, $\bar{\mu}_{\bar{\Phi}}^{r}[\alpha]$ representa os $\alpha$-níveis de um conjunto fuzzy $\bar{\mu}_{\bar{\Phi}}^{r}$. Além disso, $g_{\bar{\Phi}, 1}^{r}\left(\mathcal{S}_{\bar{\Phi}}^{1}\right)=\bar{\mu}_{\bar{\Phi}}^{r}[1] \neq \emptyset$, pois como $\left(\phi_{1}, \ldots, \phi_{n}\right) \in \mathcal{S}_{\bar{\Phi}}^{1}$, então $\phi_{1} \cdot r_{1}+\ldots+\phi_{n} \cdot r_{n} \in$ $\bar{\mu}_{\bar{\Phi}}^{r}[1]$, e, portanto, $\bar{\mu}_{\bar{\Phi}}^{r}$ é um número fuzzy.

\section{Conceitos Básicos da Teoria dos Jogos e Jogos Bayesianos}

Nesta seção, resumem-se os conceitos básicos sobre a Teoria dos Jogos [27, 35, 37, 52], em particular, sobre Jogos Bayesianos [32, 57], que é o foco deste trabalho.

Segundo Fiani [27], um jogo é "uma representação formal que permite a análise das situações em que agentes interagem entre si, agindo racionalmente". Por agentes, ou jogadores, entende-se qualquer entidade que possa tomar decisões. Os jogos são interações estratégicas, pois os jogadores se afetam mutuamente, de acordo com ações que representem suas preferências.

Existem dois tipos básicos de jogos: o jogo sequencial e o jogo simultâneo.

No jogo sequencial, cada jogador tem sua vez para agir, geralmente obedecendo a uma ordem restrita, e sua ação pode ser baseada nas ações feitas antes dele pelos outros jogadores. O comportamento estratégico faz com que cada jogador pense também nas ações futuras dos outros jogadores, que terão conhecimento de sua ação. Um exemplo clássico de jogo sequencial é o xadrez.

Quando as ações passadas são de conhecimento comum, diz-se que o jogo é de informação perfeita, como acontece na maioria dos jogos sequenciais, do contrário, diz-se que o jogo é de informação imperfeita.

Em um jogo simultâneo, todos os jogadores agem simultaneamente e sem saber de antemão as escolhas feitas pelos demais agentes; logo, trata-se de um jogo de informação imperfeita. Esse será o tipo de jogo a ser estudado nesse trabalho.

\subsection{Jogo Simultâneo com 2 Jogadores}

A forma mais usual de se modelar um jogo simultâneo é através de uma tabela, onde em cada célula são apresentadas as recompensas de cada jogador dada uma certa combinação de ações dos jogadores, conhecida como forma estratégica, como mostrado na Tabela 1 . Nesse jogo, o jogador denotado por $J_{\mathbf{A}}$ tem como opção de ação uma das estratégias no conjunto $\mathbf{A}=\left\{A_{1}, \ldots, A_{n}\right\}$, com $n \in \mathrm{N}$, e o jogador denotado por $J_{\mathbf{B}}$ pode optar pelas 


\section{Buckley $^{1}$}

Uma Abordagem para Jogos Bayesianos Fuzzy com base na Probabilidade Fuzzy de

estratégias no conjunto $\mathbf{B}=\left\{B_{1}, \ldots, B_{n^{\prime}}\right\}, \operatorname{com} n^{\prime} \in \mathrm{N}$.

Tabela 1. Forma estratégica de representação de um jogo simultâneo

\begin{tabular}{c|c|c|c|c}
\hline & \multicolumn{4}{|c}{$J_{\mathbf{B}}$} \\
\hline$J_{\mathbf{A}}$ & $B_{1}$ & $B_{2}$ & $\ldots$ & $B_{n^{\prime}}$ \\
\hline$A_{1}$ & $h_{\mathbf{A}}\left(A_{1}, B_{1}\right), h_{\mathbf{B}}\left(B_{1}, A_{1}\right)$ & $h_{\mathbf{A}}\left(A_{1}, B_{2}\right), h_{\mathbf{B}}\left(B_{2}, A_{1}\right)$ & $\ldots$ & $h_{\mathbf{A}}\left(A_{1}, B_{n^{\prime}}\right), h_{\mathbf{B}}\left(B_{n^{\prime}}, A_{1}\right)$ \\
\hline$A_{2}$ & $h_{\mathbf{A}}\left(A_{2}, B_{1}\right), h_{\mathbf{B}}\left(B_{1}, A_{2}\right)$ & $h_{\mathbf{A}}\left(A_{2}, B_{2}\right), h_{\mathbf{B}}\left(B_{2}, A_{2}\right)$ & $\ldots$ & $h\left(A_{2}, B_{n^{\prime}}\right), h_{\mathbf{B}}\left(B_{n^{\prime}}, A_{2}\right)$ \\
\hline$\vdots$ & $\vdots$ & $\vdots$ & $\vdots$ & $\vdots$ \\
\hline$A_{n}$ & $h_{\mathbf{A}}\left(A_{n}, B_{1}\right), h_{\mathbf{B}}\left(B_{1}, A_{n}\right)$ & $h_{\mathbf{A}}\left(A_{n}, B_{2}\right), h_{\mathbf{B}}\left(B_{2}, A_{n}\right)$ & $\ldots$ & $h_{\mathbf{A}}\left(A_{n}, B_{n^{\prime}}\right), h_{\mathbf{B}}\left(B_{n^{\prime}}, A_{n}\right)$ \\
\hline
\end{tabular}

Funções de recompensa $h_{\mathbf{A}}: \mathbf{A} \times \mathbf{B} \rightarrow \mathrm{R}$ e $h_{\mathbf{B}}: \mathbf{B} \times \mathbf{A} \rightarrow \mathrm{R}$ são definidas para os jogadores $J_{\mathbf{A}}$ e $J_{\mathbf{B}}$, respectivamente, tais que, para uma combinação de estratégias $\left(A_{i}, B_{j}\right)$, com $A_{i} \in \mathbf{A}$ e $B_{j} \in \mathbf{B}, h_{\mathbf{A}}\left(A_{i}, B_{j}\right)$ é a recompensa do jogador $J_{\mathbf{A}}$ ao realizar a ação $A_{i}$ dado que o jogador $J_{\mathbf{B}}$ realizou a ação $B_{j}$, assim como $h_{\mathbf{B}}\left(B_{j}, A_{i}\right)$ é recompensa do jogadores $J_{\mathrm{B}}$ pela mesma combinação de estratégias.

Um jogo simultâneo pode ser expresso através de sua matriz de recompensas

$$
R_{\mathbf{A B}}=\left[\left(h_{\mathbf{A}}\left(A_{i}, B_{j}\right), h_{\mathbf{B}}\left(B_{j}, A_{i}\right)\right)\right]_{A_{i} \in \mathbf{A}, B_{j} \in \mathbf{B}},
$$

da seguinte forma:

$$
R_{\mathbf{A B}}=\left[\begin{array}{ccc}
\left(h_{\mathbf{A}}\left(A_{1}, B_{1}\right), h_{\mathbf{B}}\left(B_{1}, A_{1}\right)\right) & \ldots & \left(h_{\mathbf{A}}\left(A_{1}, B_{n^{\prime}}\right), h_{\mathbf{B}}\left(B_{n^{\prime}}, A_{1}\right)\right) \\
\vdots & \vdots & \vdots \\
\left(h_{\mathbf{A}}\left(A_{n}, B_{1}\right), h_{\mathbf{B}}\left(B_{1}, A_{n}\right)\right) & \ldots & \left(h_{\mathbf{A}}\left(A_{n}, B_{n^{\prime}}\right), h_{\mathbf{B}}\left(B_{n^{\prime}}, A_{n}\right)\right)
\end{array}\right]
$$

onde cada elemento $\left(h_{\mathbf{A}}\left(A_{i}, B_{j}\right), h_{\mathbf{B}}\left(B_{j}, A_{i}\right)\right)$ é um par contendo as recompensas dos 2 jogadores para uma combinação de estratégias $\left(A_{i}, B_{j}\right)$, com $A_{i} \in \mathbf{A}$ e $B_{j} \in \mathbf{B}$. Ou seja, $R_{\mathrm{AB}}$ é a representação matricial da Tabela 1 .

Para se escolher a estratégia que produza o maior ganho para um dado jogador, relativa às possíveis ações dos demais jogadores, pode-se utilizar o conceito de Equilíbrio de Nash (EN). Segundo [27], "uma combinação de estratégias constitui um EN quando cada estratégia é a melhor resposta possível às estratégias dos demais jogadores, e isso é verdade para todos os jogadores".

Muitas vezes, uma recompensa em uma dada combinação de estratégias pode ser a maior possível para o Jogador $J_{\mathbf{A}}$, mas não ser interessante para o Jogador $J_{\mathbf{B}}$, logo, o Jogador $J_{\mathrm{B}}$ não vai optar por uma ação que lhe traga prejuízo, e essa combinação de estratégias não vai ser obtida. Assim, o EN, quando existe em um jogo, determina uma combinação de 
Uma Abordagem para Jogos Bayesianos Fuzzy com base na Probabilidade Fuzzy de Buckley ${ }^{1}$

ações dos jogadores que produza as recompensas mais aceitáveis para cada jogador, levando em consideração todas as ações de todos jogadores.

Para encontrar o EN, deve-se escolher, para cada jogador, qual a maior recompensa (numérica) para cada ação dos demais jogadores, e então ver se alguma combinação de recompensas foi escolhida por todos os jogadores. Em caso afirmativo, esta combinação configura um EN. Formalmente, uma combinação $\left(A_{i}, B_{j}\right)$, com $1 \leq i \leq n$ e $1 \leq j \leq n^{\prime}$, representa um EN se e somente se:

$$
\begin{aligned}
& \forall 1 \leq z \leq n, \quad \forall 1 \leq w \leq n^{\prime}: \\
& \quad h_{\mathbf{A}}\left(A_{i}, B_{j}\right) \geq h_{\mathbf{A}}\left(A_{z}, B_{j}\right) \wedge h_{\mathbf{B}}\left(B_{j}, A_{i}\right) \geq h_{\mathbf{B}}\left(B_{w}, A_{i}\right),
\end{aligned}
$$

onde $A_{z}$ e $B_{w}$ representam, respectivamente, estratégias dos jogadores $J_{\mathbf{A}}$ e $J_{\mathbf{B}}$ que não fazem parte de um EN.

Uma maneira simples de se encontrar um EN é através da análise da representação estratégica do jogo, como indicado na Tabela 1. O procedimento é encontrar, para cada coluna da tabela, quais as combinações de estratégias que retornam as maiores recompensas para o jogador $J_{\mathbf{A}}$, sendo que apenas uma combinação deve ser marcada por coluna (exceto se houver mais de uma recompensa empatada como a maior da coluna, então ambas devem ser marcadas). Em seguida, repete-se esse procedimento mas agora analisando as linhas ao invés das colunas, e levando em consideração as recompensas para o jogador $J_{\mathbf{B}}$ ao invés do jogador $J_{\mathbf{A}}$. Se uma combinação de estratégias for marcada mais de uma vez, então ela estabelece um EN.

No caso em que há mais de um EN, então outros elementos do problema modelado devem ser levados em consideração para a tomada de decisão de cada jogador. Para este trabalho, basta que se saiba encontrar o EN, caso ele exista no jogo.

\subsection{Jogo Bayesiano com 2 Jogadores}

Em um jogo simultâneo, quando um ou mais jogadores desconhecem o tipo de algum outro jogador ou até mesmo de si mesmo (isto é, suas características relevantes para o processo de decisão), então essa situação define um jogo de informação incompleta, ou jogo Bayesiano.

Nesse jogo, o jogador que não sabe o tipo do outro deve atribuir probabilidades para cada tipo possível que esse agente pode assumir. Uma vez que essas probabilidades tenham sido atribuídas (possivelmente através da opinião de especialistas no assunto), então as funções de recompensa podem ser calculadas e o jogo deixa de ter informação incompleta, tornando-se um jogo de informação imperfeita.

Consideram-se $L=\{1, \ldots, l\}$ e $M=\{1, \ldots, m\}$ os conjuntos finitos dos tipos 
possíveis que os jogadores $J_{\mathbf{A}}$ e $J_{\mathbf{B}}$ podem assumir, respectivamente. Denotam-se por $J_{\mathbf{A}}^{t}$ e $J_{\mathbf{B}}^{t^{\prime}}$ o jogador $J_{\mathbf{A}}$ do tipo específico $t \in L$ e o jogador $J_{\mathbf{B}}$ do tipo específico $t^{\prime} \in M$, respectivamente. Denotam-se por $J_{\mathbf{A}}^{L}$ e $J_{\mathbf{B}}^{M}$ os jogadores $J_{\mathbf{A}}$ e $J_{\mathbf{B}}$ cujos tipos variam nos conjuntos $L$ e $M$ respectivamente.

Um jogador $J_{\mathbf{A}}^{t}$ tem como opção de ação uma das estratégias no conjunto $\mathbf{A}=$ $\left\{A_{1}, \ldots, A_{n}\right\}$, e o jogador $J_{\mathbf{B}}^{t^{\prime}}$ pode optar pelas estratégias no conjunto $\mathbf{B}=\left\{B_{1}, \ldots, B_{n^{\prime}}\right\}$.

As recompensas vão depender dos tipos assumidos por $J_{\mathbf{A}}^{L}$ e $J_{\mathbf{B}}^{M}$, sendo então determinadas por funções de recompensa $h_{\mathbf{A}}^{t t^{\prime}}: \mathbf{A} \times \mathbf{B} \rightarrow \mathrm{R}$ e $h_{\mathbf{B}}^{t^{\prime} t}: \mathbf{B} \times \mathbf{A} \rightarrow \mathrm{R}$, para cada combinação de tipos $t \in L$ e $t^{\prime} \in M$.

Então, deve-se construir um jogo para cada combinação dos tipos em $L$ e $M$. O resultado será uma matriz $l \times m$ de jogos

$$
G_{\mathbf{A B}}^{L M}=\left[R_{\mathbf{A B}}^{t t^{\prime}}\right]_{t \in L, t^{\prime} \in M}
$$

da seguinte forma:

$$
G_{\mathbf{A B}}^{L M}=\left[\begin{array}{cccc}
R_{\mathbf{A B}}^{11} & R_{\mathbf{A B}}^{12} & \ldots & R_{\mathbf{A B}}^{1 m} \\
R_{\mathbf{A B}}^{21} & R_{\mathbf{A B}}^{22} & \ldots & R_{\mathbf{A B}}^{2 m} \\
\vdots & \vdots & \vdots & \vdots \\
R_{\mathbf{A B}}^{l 1} & R_{\mathbf{A B}}^{l 2} & \ldots & R_{\mathbf{A B}}^{l m}
\end{array}\right]
$$

onde cada entrada $R_{\mathrm{AB}}^{t t^{\prime}}$, com $t \in L$ e $t^{\prime} \in M$, denota a matriz de recompensas do jogo entre os jogadores $J_{\mathbf{A}}^{t}$ e $J_{\mathrm{B}}^{t^{\prime}}$, dada como na Eq. (29), e, portanto, tem-se que:

$$
R_{\mathbf{A} \mathbf{B}}^{t t^{\prime}}=\left[\begin{array}{ccc}
\left(h_{\mathbf{A}}^{t t^{\prime}}\left(A_{1}, B_{1}\right), h_{\mathbf{B}}^{t^{\prime} t}\left(B_{1}, A_{1}\right)\right) & \ldots & \left(h_{\mathbf{A}}^{t t^{\prime}}\left(A_{1}, B_{n^{\prime}}\right), h_{\mathbf{B}}^{t^{\prime} t}\left(B_{n^{\prime}}, A_{1}\right)\right) \\
\left(h_{\mathbf{A}}^{t t^{\prime}}\left(A_{2}, B_{1}\right), h_{\mathbf{B}}^{t^{\prime} t}\left(B_{1}, A_{2}\right)\right) & \ldots & \left(h_{\mathbf{A}}^{t t^{\prime}}\left(A_{2}, B_{n^{\prime}}\right), h_{\mathbf{B i}}^{t^{\prime} t}\left(B_{n^{\prime}}, A_{2}\right)\right) \\
\vdots & \vdots & \vdots \\
\left(h_{\mathbf{A}}^{t t^{\prime}}\left(A_{n}, B_{1}\right), h_{\mathbf{B}}^{t^{\prime} t}\left(B_{1}, A_{n}\right)\right) & \ldots & \left(h_{\mathbf{A}}^{t t^{\prime}}\left(A_{n}, B_{n^{\prime}}\right), h_{\mathbf{B}}^{t^{\prime} t}\left(B_{n^{\prime}}, A_{n}\right)\right)
\end{array}\right]
$$

onde $\mathbf{A}=\left\{A_{1}, \ldots, A_{n}\right\}$ e $\mathbf{B}=\left\{B_{1}, \ldots, B_{n^{\prime}}\right\}$ são os conjuntos de ações disponíveis para os jogadores $J_{\mathbf{A}}^{t}$ e $J_{\mathbf{B}}^{t^{\prime}}$, respectivamente.

O próximo passo é construir uma distribuição de probabilidades para os $l$ tipos possíveis de $J_{\mathbf{A}}^{L}$ e outra para os $m$ tipos possíveis de $J_{\mathbf{B}}^{M}$. Sejam $P: \wp(L) \rightarrow[0 ; 1] \mathrm{e}$ 
Uma Abordagem para Jogos Bayesianos Fuzzy com base na Probabilidade Fuzzy de Buckley ${ }^{1}$

$P^{\prime}: \wp(M) \rightarrow[0 ; 1]$ funções de probabilidade conhecidas, então $P(\{t\})=\phi_{t}$, com $t \in L$, e $P^{\prime}\left(\left\{t^{\prime}\right\}\right)=\phi_{t^{\prime}}^{\prime}$, com $t^{\prime} \in M$. Assim, $\Phi=\left\{\phi_{1}, \ldots, \phi_{l}\right\}$ e $\Phi^{\prime}=\left\{\phi_{1}^{\prime}, \ldots, \phi_{m}^{\prime}\right\}$ são as distribuições de probabilidades procuradas, e tem-se que $\sum_{t=1}^{l} \phi_{t}=1 \mathrm{e} \sum_{t^{\prime}=1}^{m} \phi_{t^{\prime}}^{\prime}=1$. O objetivo é utilizar essas probabilidades para, a partir de uma matriz de jogos determinísticos, construir um único jogo probabilístico. Este jogo será representado por uma matriz onde em cada célula se terá uma combinação de estratégias escolhidas por cada jogador $J_{\mathbf{A}}^{t}$ e cada jogador $J_{\mathbf{B}}^{t^{\prime}}$, e cujo valor da recompensa será dado ao se ponderar as probabilidades $\phi_{t}$ e $\phi_{t^{\prime}}$ atribuídas a cada tipo envolvido na combinação com os valores das recompensas contidas na respectiva matriz $R_{\mathrm{AB}}^{t \prime^{\prime}}$.

Então, as recompensas do jogo bayesiano serão obtidas através das funções

$$
h_{\mathbf{A}}^{L}: \mathbf{A}^{l} \times \mathbf{B}^{m} \rightarrow \mathrm{R}
$$

e

$$
h_{\mathbf{B}}^{M}: \mathbf{B}^{m} \times \mathbf{A}^{l} \rightarrow \mathrm{R},
$$

definidas, como a aplicação de duas médias ponderadas, por: [27]

$$
h_{\mathbf{A}}^{L}\left(A^{*}, B^{*}\right)=\sum_{t=1}^{l} \sum_{t^{\prime}=1}^{m} \phi_{t} \cdot \phi_{t^{\prime}}^{\prime} \cdot h_{\mathbf{A}}^{t t^{\prime}}\left(A^{t}, B^{t^{\prime}}\right)
$$

e

$$
h_{\mathbf{B}}^{M}\left(B^{*}, A^{*}\right)=\sum_{t^{\prime}=1}^{m} \sum_{t=1}^{l} \phi_{t} \cdot \phi_{t^{\prime}}^{\prime} \cdot h_{\mathbf{B}}^{t^{\prime} t}\left(B^{t^{\prime}}, A^{t}\right)
$$

onde $A^{*}=\left(A^{1}, \ldots, A^{l}\right)$ e $B^{*}=\left(B^{1}, \ldots, B^{m}\right)$ representam vetores que possuem, em cada posição, uma das ações dos jogadores $J_{\mathbf{A}}^{L}$ e $J_{\mathbf{B}}^{M}$, respectivamente, sendo que $A^{1}, \ldots, A^{l} \in$ $\mathbf{A}=\left\{A_{1}, \ldots, A_{n}\right\}$ e $B^{1}, \ldots, B^{m} \in \mathbf{B}=\left\{B_{1}, \ldots, B_{n^{\prime}}\right\}$.

Observe que $h_{\mathbf{A}}^{t t^{\prime}}\left(A^{t}, B^{t^{\prime}}\right)$ denota a recompensa contida na matriz $R_{\mathbf{A B}}^{t t^{\prime}}$, definida na Eq. (32), quando $J_{\mathbf{A}}^{t}$ escolhe $A^{t} \in \mathbf{A}$ e $J_{\mathbf{B}}^{t^{\prime}}$ escolhe $B^{t^{\prime}} \in \mathbf{B}$, sendo que essas escolhas são refletidas nos próprios vetores $\left(A^{1}, \ldots, A^{l}\right)$ e $\left(B^{1}, \ldots, B^{m}\right)$ utilizados para formar a combinação de estratégias investigada.

Uma vez obtidas todas as recompensas probabilísticas do jogo bayesiano, basta representá-lo na forma matricial. O resultado será uma matriz $Z_{\mathrm{AB}}$ de ordem $\left\{n^{l}\right\} \times\left\{n^{\prime m}\right\}$ que cobre todas as combinações de ações e tipos dos jogadores. Com a matriz probabilística construída, pode-se investigar a existência de um EN. O procedimento é análogo ao de um jogo simultâneo comum. 


\section{Buckley $^{1}$}

Uma Abordagem para Jogos Bayesianos Fuzzy com base na Probabilidade Fuzzy de

\section{Jogo Bayesiano Fuzzy com 2 Jogadores}

Nesta seção, introduz-se a formalização para um jogo Bayesiano fuzzy para o caso de dois jogadores. Para tanto, considera-se que a versão comum, ou crisp, do jogo Bayesiano já tenha sido desenvolvida com 2 jogadores, $J_{\mathbf{A}}^{L}$ (com $l$ tipos possíveis) e $J_{\mathbf{B}}^{M}$ (com $m$ tipos possíveis), e que já se tenha acesso à matriz de jogos $G_{\mathbf{A B}}^{L M}=\left[R_{\mathrm{AB}}^{t t^{\prime}}\right]_{t \in L, t^{\prime} \in M}$ (Eq. (31)), com todas as recompensas calculadas para todas as combinação de tipos dos jogadores em $L$ e $M$.

No entanto, algumas das probabilidades $\phi \in \Phi$ e $\phi^{\prime} \in \Phi^{\prime}$ são incertas e serão aproximadas por números fuzzy $\bar{\phi}$ e $\bar{\phi}^{\prime}$, e essas distribuições passarão a ser denotadas por $\bar{\Phi}$ e $\bar{\Phi}^{\prime}$.

De acordo com as definições de probabilidade fuzzy apresentadas na Seção 4, sabe-se que $\bar{\Phi}$ e $\bar{\Phi}^{\prime}$ devem representar distribuições finitas e discretas, mesmo que alguns de seus elementos sejam difusos. Logo, as probabilidades $\bar{\phi} \in \bar{\Phi}$ e $\bar{\phi}^{\prime} \in \bar{\Phi}^{\prime}$ devem respeitar a restrição aritmética definida.

Uma vez que todas as probabilidades fuzzy que compõem as distribuições $\bar{\Phi}$ e $\bar{\Phi}^{\prime}$ tenham sido devidamente atribuídas, deve-se ponderar essas probabilidades pelas respectivas recompensas contidas na matriz $G_{\mathbf{A B}}^{L M}=\left[R_{\mathbf{A B}}^{t t^{\prime}}\right]_{t \in L, t^{\prime} \in M}$ (Eq. (31)), utilizando a definição de média fuzzy e respeitando a restrição aritmética (Eq. (28)), para produzir a matriz definitiva $\bar{Z}_{\mathrm{AB}}$ contendo as recompensas fuzzy probabilísticas do jogo. A matriz final $\bar{Z}_{\mathrm{AB}}$ é obtida de forma análoga ao caso crisp, apenas adaptando o processo para o uso de números e operações fuzzy.

Seja $A^{*}=\left(A^{1}, \ldots, A^{l}\right)$ o vetor contendo uma combinação ações escolhidas por cada jogador $J_{\mathbf{A}}^{t}$ e $B^{*}=\left(B^{1}, \ldots, B^{m}\right)$ um vetor análogo contendo uma combinação de ações para cada jogador $J_{\mathrm{B}}^{t^{\prime}}$, como definido anteriormente. Considera-se então os conjuntos, definidos para o jogador $J_{\mathbf{A}}^{L}$ como:

$$
H_{A^{*} B^{*}}^{\mathbf{A}}=\left\{\left(h_{\mathbf{A}}^{11}\left(A^{1}, B^{1}\right), \ldots, h_{\mathbf{A}}^{1 m}\left(A^{1}, B^{m}\right)\right), \ldots,\left(h_{\mathbf{A}}^{l 1}\left(A^{l}, B^{1}\right), \ldots, h_{\mathbf{A}}^{l m}\left(A^{l}, B^{m}\right)\right)\right\},
$$

dos vetores cujas coordenadas constituem as recompensas de um jogador $J_{\mathbf{A}}^{t}$, para $t \in L$, para a combinação de vetores $A^{*}$ e $B^{*}$, quando o jogador $J_{\mathrm{B}}$ varia seu tipo $t^{\prime}$ em $M$, representado pelos jogadores $J_{\mathrm{B}}^{t^{\prime}}$. Essas recompensas são expressas pelas matrizes $R_{\mathrm{AB}}^{t t^{\prime}}$, de acordo com cada $t$ e $t^{\prime}$ correspondentes.

Em seguida, denota-se por

$$
\left.H_{A^{*} B^{*}}^{\mathbf{A}}\right|_{k}=\left(h_{\mathbf{A}}^{k 1}\left(A^{k}, B^{1}\right), \ldots, h_{\mathbf{A}}^{k m}\left(A^{k}, B^{m}\right)\right) \in H_{A^{*} B^{*}}^{\mathbf{A}}
$$

o vetor das recompensas do jogador $J_{\mathbf{A}}^{k}$, com o tipo específico $k \in L$, quando interage com 
Uma Abordagem para Jogos Bayesianos Fuzzy com base na Probabilidade Fuzzy de Buckley ${ }^{1}$

cada jogador $J_{\mathbf{B}}^{t^{\prime}}$, ou seja, quando $J_{\mathbf{B}}$ varia seu tipo $t^{\prime}$ em $M$. Agora, com base na Eq. (36), considera-se os conjuntos definidos, para $0 \leq \alpha \leq 1$, como:

$$
W_{\alpha}^{A^{*} B^{*}}=\left\{\left(g_{\bar{\Phi}^{\prime}, \alpha}^{\left.H^{*} B^{*}\right|_{1}}(e), \ldots, g_{\bar{\Phi}^{\prime}, \alpha}^{\left.H_{A^{*} B^{*}}^{\mathbf{A}_{2}}\right|_{l}}(e)\right) \mid e=\left(e_{1}, \ldots, e_{m}\right) \in \mathcal{S}_{\bar{\Phi}^{\prime}}^{\alpha}\right\},
$$

onde $\left.g_{\bar{\Phi}^{\prime}, \alpha}^{H_{A^{*} B^{*}}}\right|_{k}: \mathcal{S}_{\bar{\Phi}^{\prime}}^{\alpha} \rightarrow$ R é definida para $k \in L$ pela Eq. (27) e $\mathcal{S}_{\overline{\Phi^{\prime}}}^{\alpha}$ é dado na Eq. (23), sendo $\Phi^{\prime}=\left\{\phi_{1}^{\prime}, \ldots, \phi_{m}^{\prime}\right\}$ a distribuições de probabilidades dos $m$ tipos possíveis para o jogador $J_{\mathbf{B}}^{M}$. Salienta-se que a restrição aritmética é respeitada na definição do conjunto $W_{\alpha}^{A^{*} B^{*}}$.

Observe que, a partir da Eq. (37), para cada $k \in L$, tem-se o cálculo de uma média fuzzy relativa a $k$, cujos $\alpha$-níveis, para $0 \leq \alpha \leq 1$, são dados como:

$$
\bar{\mu}_{\bar{\Phi}^{\prime}}^{\left.H^{A^{*} B^{*}}\right|_{k}}[\alpha]=\left\{\operatorname{Proj}_{k}(w) \mid w \in W_{\alpha}^{A^{*} B^{*}}\right\},
$$

onde $\operatorname{Proj}_{k}: W_{\alpha}^{A^{*} B^{*}} \rightarrow \mathrm{R}$ é a função de projeção para a coordenada de ordem $k$ do vetor $w$, sendo a média fuzzy calculada como na Eq. (28), onde $\left.H_{A^{*} B^{*}}^{\mathbf{A}}\right|_{k}$ é dado na Eq. (36).

Finalmente, as recompensas fuzzy do jogador $J_{\mathbf{A}}^{L}$, para uma dada combinação de vetores de estratégias $\left(A^{*}, B^{*}\right)$, serão obtidas, para $0 \leq \alpha \leq 1$, através de seus $\alpha$-níveis dados como:

$$
\begin{aligned}
\bar{h}_{\mathbf{A}}^{L}\left(A^{*}, B^{*}\right)[\alpha] & =\left\{g_{\bar{\Phi}, \alpha}^{w}(e) \mid e=\left(e_{1}, \ldots, e_{l}\right) \in \mathcal{S}_{\bar{\Phi}}^{\alpha} \wedge w \in W_{\alpha}^{A^{*} B^{*}}\right\} \\
& =\bigcup_{w \in W_{\alpha}^{A^{*} B^{*}}} \bar{\mu}_{\bar{\Phi}}^{w}[\alpha]
\end{aligned}
$$

onde $\Phi=\left\{\phi_{1}, \ldots, \phi_{l}\right\}$ é a distribuições de probabilidades dos $l$ tipos possíveis para o jogador $J_{\mathbf{A}}^{L}, \bar{\mu}_{\bar{\Phi}}^{w}[\alpha]$ definem os $\alpha$-níveis da média fuzzy tal como na Eq. (28), $g_{\bar{\Phi}, \alpha}^{w}: \mathcal{S}_{\bar{\Phi}}^{\alpha} \rightarrow \mathrm{R}$ é definida pela Eq. (27), $\mathcal{S}_{\bar{\Phi}}^{\alpha}$ é dado na Eq. (23) e $W_{\alpha}^{A^{*} B^{*}}$ é definido na Eq. (37).

A Eq. (39) nada mais é do que a "fuzzificação" da Eq. (33), considerando o conceito de média fuzzy, garantindo a restrição aritmética adotada.

As recompensas do jogador $J_{\mathbf{B}}^{M}$ são obtidas de maneira análoga, como "fuzzificação" da Eq. (34). Para tanto, considere os conjuntos definidos para o jogadores $J_{\mathbf{B}}^{M}, A^{*}=$ $\left(A^{1}, \ldots, A^{l}\right)$ e $B^{*}=\left(B^{1}, \ldots, B^{m}\right)$, como:

$$
H_{B^{*} A^{*}}^{\mathbf{B}}=\left\{\left(h_{\mathbf{B}}^{11}\left(B^{1}, A^{1}\right), \ldots, h_{\mathbf{B}}^{1 l}\left(B^{1}, A^{l}\right)\right), \ldots,\left(h_{\mathbf{B}}^{m 1}\left(B^{m}, A^{1}\right), \ldots, h_{\mathbf{B}}^{m l}\left(B^{m}, A^{l}\right)\right)\right\},
$$

dos vetores cujas coordenadas constituem as recompensas de um jogador $J_{\mathrm{B}}^{t^{\prime}}$, para $t^{\prime} \in M$, quando o jogador $J_{\mathbf{A}}$ varia seu tipo $t$ em $L$, através de cada jogador $J_{\mathbf{A}}^{t}$. Da mesma forma que na Eq. (36), denota-se por

$$
\left.H_{B^{*} A^{*}}^{\mathrm{B}}\right|_{k}=\left(h_{\mathbf{B}}^{k 1}\left(B^{k}, A^{1}\right), \ldots, h_{\mathbf{B}}^{k l}\left(B^{k}, A^{l}\right)\right) \in H_{B^{*} A^{*}}^{\mathrm{B}}
$$


o vetor das recompensas do jogador $J_{\mathbf{B}}^{k}$, com o tipo específico $k \in M$, quando o jogador $J_{\mathbf{A}}$ varia seu tipo $t$ em $L$. Agora, com base na Eq. (41), considera-se os conjuntos definidos, para $0 \leq \alpha \leq 1$, como:

$$
W_{\alpha}^{B^{*} A^{*}}=\left\{\left(g_{\bar{\Phi}, \alpha}^{\left.H_{B^{*} A^{*}}^{\mathrm{B}}\right|_{1}}(e), \ldots, g_{\bar{\Phi}, \alpha}^{\left.H_{B^{*} A^{*}}^{\mathrm{B}}\right|_{m}}(e)\right) \mid e=\left(e_{1}, \ldots, e_{l}\right) \in \mathcal{S}_{\bar{\Phi}}^{\alpha}\right\},
$$

onde $g_{\bar{\Phi}, \alpha}^{\left.H_{B^{*} A^{*}}^{\mathrm{B}}\right|_{k}}: \mathcal{S}_{\bar{\Phi}}^{\alpha} \rightarrow \mathrm{R}$ é definida para $k \in M$ pela Eq. (27) e $\mathcal{S}_{\bar{\Phi}}^{\alpha}$ é dado na Eq. (23), sendo $\Phi=\left\{\phi_{1}, \ldots, \phi_{l}\right\}$ a distribuições de probabilidades dos $l$ tipos possíveis para o jogador $J_{\mathbf{A}}^{L}$.

Novamente, a partir da Eq. (42), para cada $k \in M$, tem-se o cálculo de uma média fuzzy relativa a $k$, cujos $\alpha$-níveis, para $0 \leq \alpha \leq 1$, são dados como:

$$
\bar{\mu}_{\bar{\Phi}}^{\left.H_{B^{*} A^{*}}^{\mathrm{B}}\right|_{k}}[\alpha]=\left\{\operatorname{Proj}_{k}(w) \mid w \in W_{\alpha}^{B^{*} A^{*}}\right\},
$$

onde $\operatorname{Proj}_{k}: W_{\alpha}^{B^{*} A^{*}} \rightarrow \mathrm{R}$ é a função de projeção para a coordenada de ordem $k$ do vetor $w$, sendo a média fuzzy calculada como na Eq. (28), onde $\left.H_{B^{*}}^{\mathrm{B}} A^{*}\right|_{k}$ é dado na Eq. (41).

Assim, para uma dada combinação de vetores de estratégias $\left(B^{*}, A^{*}\right)$, a expressão $\operatorname{dos} \alpha$-níveis das recompensas do jogador $J_{\mathbf{B}}^{M}$, para $0 \leq \alpha \leq 1$, sao dadas como:

$$
\begin{aligned}
\bar{h}_{\mathbf{B}}^{M}\left(B^{*}, A^{*}\right)[\alpha] & =\left\{g_{\bar{\Phi}^{\prime}, \alpha}^{w}(e) \mid e=\left(e_{1}, \ldots, e_{m}\right) \in \mathcal{S}_{\bar{\Phi}^{\prime}}^{\alpha} \wedge w \in W_{\alpha}^{B^{*} A^{*}}\right\} \\
& =\bigcup_{w \in W_{\alpha}^{B^{*} A^{*}}} \bar{\mu}_{\bar{\Phi}^{\prime}}^{w}[\alpha]
\end{aligned}
$$

onde $\Phi^{\prime}=\left\{\phi_{1}^{\prime}, \ldots, \phi_{m}^{\prime}\right\}$ é a distribuições de probabilidades dos $m$ tipos possíveis para o jogador $J_{\mathbf{B}}^{M}, \bar{\mu}_{\bar{\Phi}^{\prime}}^{w}[\alpha]$ definem os $\alpha$-níveis da média fuzzy tal como na Eq. (28), $g_{\bar{\Phi}^{\prime}, \alpha}^{w}: \mathcal{S}_{\bar{\Phi}^{\prime}}^{\alpha} \rightarrow$ R é definida pela Eq. (27), $\mathcal{S}_{\bar{\Phi}^{\prime}}^{\alpha}$ é dado na Eq. (23) e $W_{\alpha}^{B^{*} A^{*}}$ é definido na Eq. (42).

Pelo fato de algumas probabilidades atribuídas serem números fuzzy, é muito provável que algumas das recompensas da matriz de recompensas (Eq. (29)), denotada agora por $\bar{R}_{\mathrm{AB}}$, sejam fuzzy. No entanto, isso em nada altera o procedimento para se encontrar um EN, bastando que se utilize um método que ordene dois números fuzzy na Eq. (30). A escolha de qual método de ordenação se utilizará pode produzir diferentes resultados, e, portanto, deve-se escolher um que melhor se adapte ao problema modelado.

\section{Estudo de Exemplos: a Dependência da Solução da Relação de Or- dem}

Nesta Seção, discute-se um exemplo de jogo Bayesiano fuzzy, evidenciando a dependência da solução de EN da relação de ordem adotada, e mostrando que a utilização da 
Uma Abordagem para Jogos Bayesianos Fuzzy com base na Probabilidade Fuzzy de Buckley ${ }^{1}$

relação de ordem de Asmus-Dimuro [26], apresentada na Seção 3, pode ser uma boa alternativa para levar a melhores resultados.

Para fins de simplificação, será elaborado um exemplo de jogo Bayesiano crisp com apenas dois jogadores, cada um com apenas duas ações possíveis e sendo um deles com dois tipos possíveis. Em seguida, será utilizado o mesmo exemplo para o jogo Bayesiano fuzzy.

\subsection{Exemplo de Jogo Bayesiano Crisp}

Uma empresa tradicional, bem sucedida e com uma clientela bem definida, está pensando em contratar os serviços de uma nova agência de publicidade que acaba de surgir no mercado. A empresa, que será denominada jogador $J_{\mathbf{E}}$, possui duas opções de escolha: contratar $(C)$ ou não contratar $(N C)$, ou seja o conjunto de ações disponíveis para o jogador $J_{\mathbf{E}}$ é dado por $\mathbf{E}=\{C, N C\}$. Já a agência de publicidade, ou jogador $J_{\mathbf{A}}$, tem as seguintes alternativas: adotar uma campanha agressiva $(C A)$, atacando diretamente a concorrência, ou optar por uma campanha moderada $(C M)$, enaltecendo as qualidades de seu produto, e, assim, o conjunto de ações disponíveis para o jogador $J_{\mathbf{A}}$ é dado por $\mathbf{A}=\{C A, C M\}$.

O jogador $J_{\mathbf{A}}$ conhece bem as características do jogador $J_{\mathbf{E}}$ (a empresa possui já uma reputação conquistada por seus anos de atividade) e não terá problemas para tomar a decisão mais racional levando em consideração todas as possibilidades de ambos os jogadores. No entanto, o jogador $J_{\mathbf{E}}$ não conhece o tipo do jogador $J_{\mathbf{A}}$, pois a agência é nova e pouco se sabe a respeito de sua abordagem. Assim, o jogador $J_{\mathbf{A}}$ pode variar seu tipo no conjunto $L=\{o$ (ousado), $c$ (classico) $\}$, passando a ser denotado por $J_{\mathbf{A}}^{L}$.

A partir deste momento, o jogo Bayesiano é transformado em um jogo de informação imperfeita. São construídas então duas tabelas de jogos, uma para cada tipo possível que o jogador $J_{\mathbf{A}}^{L}$ possa assumir. Primeiro, supõe-se que a agência publicitária tenha caráter ousado (tipo $o$ ), utilizando elementos que provoquem reações emocionais fortes em suas propagandas, e, neste caso, este jogador será denotado por $J_{\mathbf{A}}^{o}$.

De acordo com os especialistas da empresa contratante (jogador $J_{\mathbf{E}}$ ), as recompensas dessa situação estratégica com esse tipo de agência se dá através da Tabela 2, onde, em cada célula $i j$, o valor da esquerda representa a recompensa do jogador $J_{\mathbf{A}}^{o}$ e o valor da direita é a recompensa do jogador $J_{\mathbf{E}}$, para a dada combinação de estratégias.

Assim, a Tabela 2 representa a matriz de recompensas $R_{\mathbf{A}^{\circ} \mathbf{E}}$ do jogo entre o jogador $J_{\mathbf{A}}^{o}$ (jogador $A$ do tipo $o$ ) e o jogador $J_{\mathbf{E}}$, que não varia seu tipo. Nesse caso, se estabeleceria um EN na combinação de estratégias

$$
(C A, N C) \text {, }
$$

ou seja, as decisões mais racionais seriam a agência apresentar uma campanha agressiva 
Tabela 2. Recompensas do jogo considerando a agência do tipo $o$ (ousado)

\begin{tabular}{c|c|c}
\hline$R_{\mathbf{A}^{\circ} \mathbf{E}}$ & \multicolumn{2}{|c}{$J_{\mathbf{E}}$} \\
\hline$J_{\mathbf{A}}^{o}$ & $C$ & $N C$ \\
\hline$C A$ & $2,-2$ & 1,0 \\
\hline$C M$ & $-1,1$ & $0,-1$ \\
\hline
\end{tabular}

(o que combina com seu tipo) e a empresa não contratar os serviços dessa agência, pois sua clientela bem estabelecida poderia ficar chocada com a campanha e sua reputação ser abalada. A agência ousada, mesmo não sendo contratada, ainda teria um ganho pelo fato de sua campanha mesmo não sendo adotada, talvez chamasse a atenção de outros contratantes.

Agora, supõe-se que a agência tenha caráter clássico (tipo $c$ ), se concentrando mais nos aspectos lúdicos em suas propagandas, e assim este jogador passa a ser denotado por $J_{\mathbf{A}}^{c}$. Em uma situação estratégica com esse jogador, os especialistas da empresa (jogador $J_{\mathbf{E}}$ ) estabelecem as recompensas contidas na Tabela 3 . Esta tabela representa a matriz $R_{\mathbf{A}^{c} \mathbf{E}}$ contendo as recompensas do jogo entre os jogadores $J_{\mathbf{A}}^{c}$ e $J_{\mathbf{E}}$.

Tabela 3. Recompensas do jogo considerando a agência do tipo $c$ (clássico)

\begin{tabular}{c|c|c}
\hline$R_{\mathbf{A}^{c} \mathbf{E}}$ & \multicolumn{2}{|c}{$J_{\mathbf{E}}$} \\
\hline$J_{\mathbf{A}}^{c}$ & $C$ & $N C$ \\
\hline$C A$ & $-2,2$ & $-1,0$ \\
\hline$C M$ & 2,2 & $0,-1$ \\
\hline
\end{tabular}

Observa-se que agora o EN se dá na combinação

$$
(C M, C),
$$

significando que a escolha mais racional seria a agência apresentar uma campanha moderada e a empresa contratar os serviços da agência, pela compatibilidade com o seu público-alvo.

O problema é justamente o fato do jogador $J_{\mathbf{E}}$ não saber em qual dos dois jogos ele está inserido. Então, para se obter apenas uma tabela de recompensas, e assim tentar encontrar um único EN, estabelece-se que um pseudo-jogador (sem recompensas), a Natureza, vai escolher aleatoriamente qual o tipo do jogador $J_{\mathbf{A}}^{L}$, atribuindo-se uma probabilidade $p$ da agência ser do tipo $o$, e probabilidade $q=(1-p)$ de ser do tipo $c$ (pois a soma dos probabilidades deve ser igual a 1 ).

As recompensas (tanto do jogador $J_{\mathbf{E}}$ quanto do jogador $J_{\mathbf{A}}^{L}$ ) serão obtidas através 
Uma Abordagem para Jogos Bayesianos Fuzzy com base na Probabilidade Fuzzy de Buckley ${ }^{1}$

do cálculo do valor esperado das recompensas para cada combinação de estratégias, levando em consideração os dois tipos possíveis do jogador $J_{\mathbf{A}}^{L}$, bem como as probabilidades desse jogador ser de cada tipo em $L$, como apresentado nas equações (33) e (34). Para melhor entendimento do processo de obtenção desses valores, serão construídas tabelas intermediárias explicitando separadamente as recompensas de cada jogador.

As tabelas de um jogo Bayesiano incluem todos os tipos possíveis que cada agente pode assumir. O Jogador $J_{\mathbf{E}}$ só possui um tipo, então nada se altera em sua representação. Como o Jogador $J_{\mathbf{A}}^{L}$ possui dois tipos possíveis em $L=\{o, c\}$, então suas ações serão representadas por meio de pares, compostos pelas ações de cada tipo como se fossem dois jogadores. Finalmente, ponderam-se as recompensas obtidas nas Tabelas 2 e 3 pelas probabilidades de acordo com o tipo que cada uma dessas tabelas representa, e somam-se esses resultados.

Na Tabela 4, são apresentadas apenas as recompensas da agência (jogador $J_{\mathbf{A}}^{L}$ ). Nos pares de ações do jogador $J_{\mathbf{A}}^{L}$, o valor da esquerda corresponde à ação do tipo $o$ e valor da direita à ação do tipo $c$.

Tabela 4. Recompensas probabilísticas do jogador $J_{\mathbf{A}}^{L}$

\begin{tabular}{c|c|c}
\hline & \multicolumn{2}{|c}{$J_{\mathbf{E}}$} \\
\hline$J_{\mathbf{A}}^{o}, J_{\mathbf{A}}^{c}$ & $C$ & $N C$ \\
\hline$C A, C A$ & $4 p-2$ & $2 p-1$ \\
\hline$C A, C M$ & 2 & $p$ \\
\hline$C M, C A$ & $p-2$ & $p-1$ \\
\hline$C M, C M$ & $2-3 p$ & 0 \\
\hline
\end{tabular}

Por exemplo, na Tabela 4, a primeira célula da primeira linha à esquerda, correspondente à combinação $((C A, C A), C)$, mostra o valor esperado das recompensas do jogador $J_{\mathbf{A}}^{L}$ para o caso de $J_{\mathbf{A}}^{o}$ e $J_{\mathbf{A}}^{c}$ escolherem a ação $C A$ e do jogador $J_{E}$ escolher a ação $C$. Da Tabela 2, obtém-se que $J_{\mathbf{A}}^{o}$ possui recompensa 2 para a combinação de ações $(C A, C)$, e, da Tabela 3, extrai-se que $J_{\mathrm{A}}^{c}$ possui recompensa -2 para essa mesma combinação. Ponderando a recompensa de $J_{\mathbf{A}}^{o}$ (valor 2) por sua probabilidade $p$ e somando com a recompensa de $J_{\mathbf{A}}^{c}$ (valor -2 ) ponderada por sua probabilidade $q=1-p$, obtém-se a expressão

$$
2 p+(-2)(1-p)=4 p-2 .
$$

As demais recompensas da Tabela 4 são calculadas da mesma forma. Observe que, na Tabela 4, para cada combinação de estratégias $\left(\left(A_{i}, A_{i}^{\prime}\right), E_{j}\right), \operatorname{com} A_{i}, A_{i}^{\prime} \in \mathbf{A}$ e $E_{j} \in \mathbf{E}$, a recom- 


\section{Buckley $^{1}$}

Uma Abordagem para Jogos Bayesianos Fuzzy com base na Probabilidade Fuzzy de

pensa esperada do jogador $J_{\mathbf{A}}^{L}$ é calculada como:

$$
p \cdot h_{\mathbf{A}}^{o-}\left(A_{i}, E_{j}\right)+q \cdot h_{\mathbf{A}}^{c-}\left(A_{i}^{\prime}, E_{j}\right),
$$

onde $p$ e $q$ são as probabilidades do jogador $J_{\mathbf{A}}^{L}$ ser do tipo $o$ ou $c$, respectivamente, $h_{\mathbf{A}}^{o-}$ e $h_{\mathbf{A}}^{c-}$ são as funções de recompensa para o jogador $J_{\mathbf{A}}^{L}$ ao assumir o tipo $o$ ou $c$, respectivamente (ver Tabelas 2 e 3$)^{7}$

Na Tabela 5, estão apenas as recompensas referentes ao Jogador $J_{\mathbf{E}}$, calculadas da mesma maneira que as recompensas da Tabela 4. Assim, para cada combinação de estratégias $\left(\left(A_{i}, A_{i}^{\prime}\right), E_{j}\right), \operatorname{com} A_{i}, A_{i}^{\prime} \in \mathbf{A}$ e $E_{j} \in \mathbf{E}$, a recompensa esperada do jogador $J_{\mathbf{E}}$ é calculada como:

$$
p \cdot h_{\mathbf{E}}^{-o}\left(E_{j}, A_{i}\right)+q \cdot h_{\mathbf{E}}^{-c}\left(E_{j}, A_{i}^{\prime}\right),
$$

onde $p$ e $q$ são as probabilidades do jogador $J_{\mathbf{A}}^{L}$ ser do tipo $o$ ou $c$, respectivamente, $h_{\mathbf{E}}^{-o}$ e $h_{\mathbf{E}}^{-c}$ são as funções de recompensa para o jogador $J_{\mathbf{E}}$ se o jogador $J_{\mathbf{A}}^{L}$ assume o tipo $o$ ou $c$, respectivamente (ver Tabelas 2 e 3 ).

Tabela 5. Recompensas probabilísticas do jogador $J_{\mathbf{E}}$

\begin{tabular}{c|c|c}
\hline & \multicolumn{2}{|c}{$J_{\mathbf{E}}$} \\
\hline$J_{\mathbf{A}}^{o}, J_{\mathbf{A}}^{c}$ & $C$ & $N C$ \\
\hline$C A, C A$ & $2-4 p$ & 0 \\
\hline$C A, C M$ & $2-4 p$ & $p-1$ \\
\hline$C M, C A$ & $2-p$ & $-p$ \\
\hline$C M, C M$ & $-p+2$ & -1 \\
\hline
\end{tabular}

Para se obter a tabela final que representa as recompensas dos dois jogadores, basta compor os resultados das Tabelas 4 e 5, onde os valores da esquerda representarão as recompensas do jogador $J_{\mathbf{A}}^{L}$ e os valores da direita as recompensas do jogador $J_{\mathbf{E}}$. O resultado está na Tabela 6.

Uma vez que se tenha uma tabela com as recompensas de ambos os jogadores em função de $p$ (Tabela 6), basta atribuir um valor para $p$ (chance da agência ser do tipo $o$ ) para então poder procurar a existência de um EN. Esse valor possivelmente vai ser estimado pelos especialistas da empresa, onde $p=0.5$ traduziria uma situação de incerteza total. Observa-se que, se $p=1$, as recompensas serão exatamente as mesmas da Tabela 2, da mesma forma que $p=0$ retornaria recompensas iguais às da Tabela 3.

\footnotetext{
${ }^{7}$ Quando um dos jogadores não varia seu tipo, representa-se a combinação de tipos " $t t^{\prime}$ " dos dois jogadores por " $t-$ " ou " $-t$ ", onde $t$ representa o tipo variável.
} 
Uma Abordagem para Jogos Bayesianos Fuzzy com base na Probabilidade Fuzzy de Buckley ${ }^{1}$

Tabela 6. Recompensas do jogo Bayesiano Crisp

\begin{tabular}{c|c|c}
\hline$R_{\mathbf{A E}}^{L-}$ & \multicolumn{2}{|c}{$J_{\mathbf{E}}$} \\
\hline$J_{\mathbf{A}}^{o}, J_{\mathbf{A}}^{c}$ & $C$ & $N C$ \\
\hline$C A, C A$ & $4 p-2,2-4 p$ & $2 p-1,0$ \\
\hline$C A, C M$ & $2,2-4 p$ & $p, p-1$ \\
\hline$C M, C A$ & $p-2,2-p$ & $p-1,-p$ \\
\hline$C M, C M$ & $2-3 p,-p+2$ & $0,-1$ \\
\hline
\end{tabular}

Supondo que os especialistas da empresa estimassem uma probabilidade de $70 \%$ da agência ser ousada (i.e., $p=0.7$ ), obtém-se os valores da Tabela 7. Um jogo com essas recompensas possui um EN na combinação de ações $((C A, C M), N C)$, ou seja, com uma chance de $70 \%$ da agência de publicidade ser ousada, a opção mais racional para empresa é não contratar o serviço dessa agência.

Tabela 7. Recompensas do jogo Bayesiano para $p=0.7$

\begin{tabular}{c|c|c}
\hline$R_{\mathbf{A E}}^{L-}$ & \multicolumn{2}{|c}{$J_{\mathbf{E}}$} \\
\hline$J_{\mathbf{A}}^{o}, J_{\mathbf{A}}^{c}$ & $C$ & $N C$ \\
\hline$C A, C A$ & $0.8,-0.8$ & $0.4,0$ \\
\hline$C A, C M$ & $2,-0.8$ & $0.7,-0.3$ \\
\hline$C M, C A$ & $-1.3,1.3$ & $-0.3,-0.7$ \\
\hline$C M, C M$ & $-0.1,1.3$ & $0,-1$ \\
\hline
\end{tabular}

Fica claro que, além das recompensas dos dois jogos em que os tipos de $J_{\mathbf{A}}^{L}$ são definidos, contidas nas Tabelas 2 e 3, o valor das probabilidades $p$ e $q$ se mostram fundamentais para que as recompensas do jogo sejam estimadas. Entretanto, em muitos casos, essas probabilidades são difíceis de serem estimadas com precisão. No caso desse exemplo, talvez os especialistas discordassem entre si quanto ao valor a ser atribuído a $p$, e por conseguinte, a $q$. Para contornar esse tipo de impasse, e modelar a imprecisão contida na atribuição da probabilidade, agora o jogo será reformulado substituindo $p$ por $\bar{p}$ e $q$ por $\bar{q}$ (probabilidades fuzzy) e passará a ser um jogo Bayesiano fuzzy, como apresentado na seção a seguir. 


\subsection{Exemplo de Jogo Bayesiano Fuzzy}

Levando em consideração que já se tem acesso aos valores das Tabelas 2 e 3 (matrizes $R_{\mathbf{A}^{\circ} \mathbf{E}}$ e $R_{\mathbf{A}^{c} \mathbf{E}}$, respectivamente), agora é preciso definir como gerar as recompensas em função das probabilidades fuzzy $\bar{p}$ e $\bar{q}$.

O conjunto dos tipos possíveis do jogador $J_{\mathbf{A}}^{L}$ é dado por $L=\{o, c\}$, com subconjuntos não vazios $Y=\{o\}$ e $Z=\{c\}$. As probabilidades fuzzy devem ser atribuídas de forma que $\bar{p}=\bar{P}(Y), \bar{q}=\bar{P}(Z)$ e $\bar{P}(L)=\bar{P}(Y \cup Z)=1$, respeitando a restrição aritmética. Tem-se então a distribuição de probabilidades $(X, \bar{P})$, representada pelo conjunto $\bar{\Phi}=\{\bar{p}, \bar{q}\}$.

Uma vez que se tenha estabelecido as probabilidades fuzzy $\bar{p}$ e $\bar{q}$, deve-se produzir as recompensas através do cálculo do valor esperado, utilizando a definição de média fuzzy com as recompensas contidas nas Tabelas 2 e 3 . Considera-se $A^{*}$ como um vetor contendo a combinação de ações escolhidas por $J_{\mathbf{A}}^{o}$ e $J_{\mathbf{A}}^{c}$, e $E^{*}$ como o vetor unitário contendo a ação escolhida por $J_{\mathbf{E}}$. Assim, tem-se, pela Eq. (35), que o conjunto dos vetores (unitários, neste caso) das recompensas do jogador $J_{\mathbf{A}}^{L}$ é dado como:

$$
H_{A^{*} E^{*}}^{\mathbf{A}}=\left\{\left(h_{\mathbf{A}}^{o-}\left(A^{o}, E^{-}\right)\right),\left(h_{\mathbf{A}}^{c-}\left(A^{c}, E^{-}\right)\right)\right\} .
$$

Agora, com base na notação da Eq. (36) e na Eq. (37), tem-se, para $0 \leq \alpha \leq 1$, que:

$$
W_{\alpha}^{A^{*} E^{*}}=\left\{\left(g_{\bar{\Phi}^{\prime}, \alpha}^{\left(h_{\mathbf{A}}^{o-}\left(A^{o}, E^{-}\right)\right)}(1), g_{\bar{\Phi}^{\prime}, \alpha}^{\left(h_{\mathbf{A}}^{c-}\left(A^{c}, E^{-}\right)\right)}(1)\right)\right\}=\left\{\left(h_{\mathbf{A}}^{o-}\left(A^{o}, E^{-}\right), h_{\mathbf{A}}^{c-}\left(A^{c}, E^{-}\right)\right)\right\},
$$

onde $g_{\bar{\Phi}^{\prime}, \alpha}^{\left(h_{\mathrm{A}}^{o-}\left(A^{o}, E^{-}\right)\right)}, g_{\bar{\Phi}^{\prime}, \alpha}^{\left(h_{\mathrm{A}}^{c-}\left(A^{c}, E^{-}\right)\right)}: \mathcal{S}_{\bar{\Phi}^{\prime}}^{\alpha} \rightarrow \mathrm{R}$ é definida pela Eq. (27) e $\mathcal{S}_{\bar{\Phi}^{\prime}}^{\alpha}$ é dado na Eq. (23), sendo $\Phi^{\prime}=\{\overline{1}\}$, uma vez que o jogador $J_{\mathbf{E}}$ não varia seu tipo.

Finalmente, pela Eq. (39), as recompensas fuzzy do jogador $J_{\mathbf{A}}^{L}$, para uma dada combinação de vetores de estratégias $\left(A^{*}, E^{*}\right)$, serão obtidas, para $0 \leq \alpha \leq 1$, através de seus $\alpha$-níveis dados como:

$$
\begin{aligned}
\bar{h}_{\mathbf{A}}^{L}\left(A^{*}, E^{*}\right)[\alpha] & =\left\{g_{\bar{\Phi}, \alpha}^{w}(e) \mid e=\left(e_{o}, e_{c}\right) \in \mathcal{S}_{\bar{\Phi}}^{\alpha} \wedge w \in W_{\alpha}^{A^{*} E^{*}}\right\} \\
& =\left\{g_{\bar{\Phi}, \alpha}^{\left(h_{\mathbf{A}}^{o-}\left(A^{o}, E^{-}\right), h_{\mathbf{A}}^{c-}\left(A^{c}, E^{-}\right)\right)}(e) \mid e=\left(e_{o}, e_{c}\right) \in \mathcal{S}_{\bar{\Phi}}^{\alpha}\right\} \\
& =\bar{\mu}_{\bar{\Phi}}^{\left(h_{\mathbf{A}}^{o-}\left(A^{o}, E^{-}\right), h_{\mathbf{A}}^{c-}\left(A^{c}, E^{-}\right)\right)}[\alpha]
\end{aligned}
$$

onde $\Phi=\{\bar{p}, \bar{q}\}$ é a distribuições de probabilidades dos 2 tipos possíveis para o jogador $J_{\mathbf{A}}^{L}, \bar{\mu}_{\bar{\Phi}}^{\left(h_{\mathbf{A}}^{o-}\left(A^{o}, E^{-}\right), h_{\mathbf{A}}^{c-}\left(A^{c}, E^{-}\right)\right)}[\alpha]$ definem os $\alpha$-níveis da média fuzzy tal como na Eq. (28), $g_{\bar{\Phi}, \alpha}^{\left(h_{\mathrm{A}}^{o-}\left(A^{o}, E^{-}\right), h_{\mathrm{A}}^{c-}\left(A^{c}, E^{-}\right)\right)}: \mathcal{S}_{\bar{\Phi}}^{\alpha} \rightarrow$ R é definida pela Eq. (27), $\mathcal{S}_{\bar{\Phi}}^{\alpha}$ é dado na Eq. (23) e $W_{\alpha}^{A^{*} E^{*}}$ é definido na Eq. (50). 
Uma Abordagem para Jogos Bayesianos Fuzzy com base na Probabilidade Fuzzy de Buckley ${ }^{1}$

Segue que:

$$
\bar{h}_{\mathbf{A}}^{L}\left(A^{*}, E^{*}\right)[\alpha]=\left\{h_{\mathbf{A}}^{o-}\left(A^{o}, E^{-}\right) \cdot e_{o}+h_{\mathbf{A}}^{c-}\left(A^{c}, E^{-}\right) \cdot e_{c} \mid e=\left(e_{o}, e_{c}\right) \in \mathcal{S}_{\bar{\Phi}}^{\alpha}\right\},
$$

lembrando que $\mathcal{S}_{\bar{\Phi}}^{\alpha}$ é o conjunto contendo todos os valores crisp $e_{o}$ e $e_{c}$ pertencentes aos $\alpha$ níveis $\bar{p}[\alpha]$ e $\bar{q}[\alpha]$, respectivamente, satisfazendo a restrição aritmética. Ou seja, na Eq. (52), considera-se o cálculo da valor esperado para cada $e_{o} \in \bar{p}[\alpha]$ e $e_{c} \in \bar{q}[\alpha]$, onde $e_{o}+e_{c}=$ 1 , como estabelecido pela restrição aritmética. Logo, como $e_{c}=1-e_{o}$, as expressões analíticas das médias calculadas serão semelhantes àquelas contidas na Tabela 6 . Assim, na Eq. (52), tem-se o conjunto dos resultados de todos os valores esperados possíveis, de forma que $e_{o}+e_{c}=1$, formando um intervalo que representa justamente os $\alpha$-níveis da média fuzzy calculada na Eq. (51).

Com um raciocínio análogo, e considerando que o jogador $J_{\mathbf{E}}$ não varia seu tipo, obtém-se, pela Eq. (35), o conjunto formado por apenas um vetor de recompensas deste jogador, que é dado como:

$$
H_{E^{*} A^{*}}^{\mathbf{E}}=\left\{\left(h_{\mathbf{E}}^{-o}\left(E^{-}, A^{o}\right), h_{\mathbf{E}}^{-c}\left(E^{-}, A^{c}\right)\right)\right\} .
$$

Agora, com base na notação da Eq. (36) e na Eq. (37), tem-se, para $0 \leq \alpha \leq 1$, que:

$$
\begin{aligned}
W_{\alpha}^{E^{*} A^{*}} & =\left\{\left(g_{\bar{\Phi}, \alpha}^{H_{E^{*}}^{\mathrm{E}} A^{*}} \mid-(e)\right) \mid e=\left(e_{o}, e_{c}\right) \in \mathcal{S}_{\bar{\Phi}}^{\alpha}\right\} \\
& =\left\{\left(g_{\bar{\Phi}, \alpha}^{\left(h_{\mathrm{E}}^{-o}\left(E^{-}, A^{o}\right), h_{\mathbf{E}}^{-c}\left(E^{-}, A^{c}\right)\right)}(e)\right) \mid e=\left(e_{o}, e_{c}\right) \in \mathcal{S}_{\bar{\Phi}}^{\alpha}\right\} \\
& =\left\{\left(h_{\mathbf{E}}^{-o}\left(E^{-}, A^{o}\right) \cdot e_{o}+h_{\mathbf{E}}^{-c}\left(E^{-}, A^{c}\right) \cdot e_{c}\right) \mid\left(e_{o}, e_{c}\right) \in \mathcal{S}_{\bar{\Phi}}^{\alpha}\right\},
\end{aligned}
$$

onde $g_{\bar{\Phi}, \alpha}^{\left.H_{i j}^{\mathrm{E}}\right|_{-}}: \mathcal{S}_{\bar{\Phi}}^{\alpha} \rightarrow \mathrm{R}$ é definida pela Eq. (27) e $\mathcal{S}_{\bar{\Phi}}^{\alpha}$ é dado na Eq. (23), sendo $\Phi=\{\bar{p}, \bar{q}\}$, uma vez que o jogador $J_{\mathbf{A}}^{L}$ varia seu tipo em $L$ de acordo com $\Phi$.

Assim, pela Eq. (39), as recompensas fuzzy do jogador $J_{\mathbf{E}}$, para uma dada combinação de vetores de estratégias $\left\{E^{*}, A^{*}\right\}$, serão obtidas, para $0 \leq \alpha \leq 1$, através de seus $\alpha$-níveis dados como:

$$
\begin{aligned}
\bar{h}_{\mathbf{E}}\left(E^{*}, A^{*}\right)[\alpha] & =\left\{g_{\bar{\Phi}^{\prime}, \alpha}^{w}(1) \mid w \in W_{\alpha}^{E^{*} A^{*}}\right\} \\
& =\left\{g_{\bar{\Phi}^{\prime}, \alpha}^{\left(h_{\mathbf{E}}^{-o}\left(E^{-}, A^{o}\right) \cdot e_{o}+h_{\mathbf{E}}^{-c}\left(E^{-}, A^{c}\right) \cdot e_{c}\right)}(1) \mid\left(e_{o}, e_{c}\right) \in \mathcal{S}_{\bar{\Phi}^{\alpha}}^{\alpha}\right\} \\
& =\bigcup_{\left(e_{o}, e_{c}\right) \in \mathcal{S}_{\bar{\Phi}}^{\alpha}} \bar{\mu}_{\bar{\Phi}^{\prime}}^{\left(h_{\mathbf{E}}^{-o}\left(E^{-}, A^{o}\right) \cdot e_{o}+h_{\mathbf{E}}^{-c}\left(E^{-}, A^{c}\right) \cdot e_{c}\right)}[\alpha]
\end{aligned}
$$

onde $\Phi^{\prime}=\{1\}$, pois o jogador $J_{\mathbf{E}}$ não varia seu tipo, $\bar{\mu}_{\bar{\Phi}^{\prime}}^{\left(h_{\mathbf{E}}^{-o}\left(E^{-}, A^{o}\right) \cdot e_{o}+h_{\mathbf{E}}^{-c}\left(E^{-}, A^{c}\right) \cdot e_{c}\right)}[\alpha]$ definem os $\alpha$-níveis da média fuzzy tal como na Eq. (28), $g_{\bar{\Phi}^{\prime}, \alpha}^{\left(h_{\mathrm{E}}^{-o}\left(E^{-}, A^{o}\right) \cdot e_{o}+h_{\mathbf{E}}^{-c}\left(E^{-}, A^{c}\right) \cdot e_{c}\right)}$ : $\mathcal{S}_{\bar{\Phi}^{\prime}}^{\alpha} \rightarrow$ R é definida pela Eq. (27), $\mathcal{S}_{\bar{\Phi}^{\prime}}^{\alpha}$ é dado na Eq. (23) e $W_{\alpha}^{E^{*}} A^{*}$ é definido na Eq. (54). 
Uma Abordagem para Jogos Bayesianos Fuzzy com base na Probabilidade Fuzzy de

Segue que:

$$
\bar{h}_{\mathbf{E}}\left(E^{*}, A^{*}\right)[\alpha]=\left\{h_{\mathbf{E}}^{-o}\left(E^{-}, A^{o}\right) \cdot e_{o}+h_{\mathbf{E}}^{-c}\left(E^{-}, A^{c}\right) \cdot e_{c} \mid\left(e_{o}, e_{c}\right) \in \mathcal{S}_{\bar{\Phi}}^{\alpha}\right\},
$$

onde $\mathcal{S}_{\bar{\Phi}}^{\alpha}$ é o conjunto contendo todos os valores crisp $e_{o}$ e $e_{c}$ pertencentes aos $\alpha$-níveis $\bar{p}[\alpha]$ e $\bar{q}[\alpha]$, respectivamente, satisfazendo a restrição aritmética.

Para analisar o comportamento do jogo quando se atribuem números fuzzy para as probabilidades $\bar{p}$ e $\bar{q}$, supõe-se que os especialistas da empresa produziram o número fuzzy triangular

$$
\bar{p}=(0.6 / 0.7 / 0.8)
$$

para modelar a noção de que probabilidade fuzzy da agência ser do tipo ousado é "em torno de" $70 \%$. Logo os $\alpha$-níveis de $\bar{p}$ são calculados como

$$
\bar{p}[\alpha]=[0.6+0.1 \alpha, 0.8-0.1 \alpha] .
$$

Por conseguinte, tem-se que

$$
\bar{q}=(0.2 / 0.3 / 0.4),
$$

ou seja, a agência tem chance "em torno de" $30 \%$ de ser do tipo clássico, e assim, tem-se os $\alpha$-níveis dados como

$$
\bar{q}[\alpha]=[0.2+0.1 \alpha, 0.4-0.1 \alpha] .
$$

Esses valores, além de intuitivamente parecerem coerentes, respeitam a restrição aritmética.

Calculando as médias fuzzy para $\bar{p}=(0.6 / 0.7 / 0.8)$ e $\bar{q}=(0.2 / 0.3 / 0.4)$, obtém-se a Tabela 8 com os valores das recompensas fuzzy de ambos os jogadores.

Tabela 8. Recompensas fuzzy do jogo bayesiano fuzzy para $\bar{p}=(0.6 / 0.7 / 0.8)$

\begin{tabular}{c|c|c}
\hline $\bar{R}_{\mathbf{A E}}^{L-}$ & $C$ & $J_{\mathbf{E}}$ \\
\hline$J_{\mathbf{A}}^{o}, J_{\mathbf{A}}^{c}$ & $(0.4 / 0.8 / 1.2),(-1.2 /-0.8 /-0.4)$ & $(0.2 / 0.4 / 0.6), 0$ \\
\hline$C A, C A$ & $2,(-1.2 /-0.8 /-0.4)$ & $(0.6 / 0.7 / 0.8),(-0.4 /-0.3 /-0.2)$ \\
\hline$C A, C M$ & $(-1.4 /-1.3 /-1.2),(1.2 / 1.3 / 1.4)$ & $(-0.4 /-0.3 /-0.2),(-0.8 /-0.7 /-0.6)$ \\
\hline$C M, C A$ & $(-0.4 /-0.1 / 0.2),(1.2 / 1.3 / 1.4)$ & $0,-1$ \\
\hline$C M, C M$ &
\end{tabular}

Utilizando as expressões da Tabela 6 para o cálculo de cada média fuzzy, basta substituir em $p$ os extremos de $\bar{p}[\alpha]$ para se obter o intervalo $\left[m_{1}, m_{2}\right]$ que representa a média $\bar{\mu}_{\bar{\Phi}}^{\left(h_{\mathbf{A}}^{o-}\left(A^{o}, E^{-}\right), h_{\mathbf{A}}^{c-}\left(A^{c}, E^{-}\right)\right)}[\alpha]$. Por exemplo, na primeira célula à esquerda da Tabela 6 , que 
Uma Abordagem para Jogos Bayesianos Fuzzy com base na Probabilidade Fuzzy de Buckley $^{1}$

representa a combinação $((C A, C A), C)$, na recompensa referente ao jogador $J_{\mathbf{A}}^{L}$, ao se substituir $p$ pelo extremo esquerdo de $\bar{p}[\alpha]$, dado como $0.6+0,1 \alpha$, se obtém

$$
m_{1}=4(0.6+0.1 \alpha)-2=0.4+0.4 \alpha,
$$

e ao se substituir $p$ pelo extremo direito de $\bar{p}[\alpha]$, dado como $0.8-0.1 \alpha$, se obtém

$$
m_{2}=4(0.8-0.1 \alpha)-2=1.2-0.4 \alpha .
$$

Ou seja,

$$
\left[m_{1}, m_{2}\right]=[0.4+0.4 \alpha, 1.2-0.4 \alpha],
$$

e esses são os $\alpha$-níveis do número fuzzy $(0.4 / 0.8 / 1.2)$, como apresentado na primeira célula à esquerda da Tabela 8 . Os outros resultados foram obtidos de maneira análoga.

Agora que foram obtidas as recompensas de ambos os jogadores, resta investigar a existência de um EN. O procedimento será o mesmo utilizado anteriormente, apenas levando em consideração os métodos escolhido neste trabalho para se ordenar números fuzzy. Neste exemplo, não há qualquer dúvida de que o equilíbrio ocorre na combinação

$$
((C A, C M), N C),
$$

pois todos os números fuzzy encontrados na Tabela 8 puderam ser comparados através da relação de ordem definida pela Eq. (9).

No entanto, mesmo que a relação de ordem definida pela Eq. (9) seja bastante simples e de fácil aplicação, nem sempre ela se mostra suficiente para apontar qual o maior entre dois números fuzzy, pois se trata de uma relação de ordem parcial, mas não total. Por exemplo, para esse mesmo jogo, ao se atribuir $\bar{p}=(0.5 / 0.6 / 0.7)$ e $\bar{q}=(0.3 / 0.4 / 0.5)$, obtém-se a seguinte tabela de recompensas:

Tabela 9. Recompensas fuzzy do jogo bayesiano fuzzy para $\bar{p}=(0.5 / 0.6 / 0.7)$

\begin{tabular}{c|c|c}
\hline $\bar{R}_{\mathbf{A E}}^{L-}$ & $C$ & $J_{\mathbf{E}}$ \\
\hline$J_{\mathbf{A}}^{o}, J_{\mathbf{A}}^{c}$ & $(0 / 0.4 / 0.8),(-0.8 /-0.4 / 0)$ & $(0 / 0.2 / 0.4), 0$ \\
\hline$C A, C A$ & $2,(-0.8 /-0.4 / 0)$ & $(0.5 / 0.6 / 0.7),(-0.5 /-0.4 /-0.3)$ \\
\hline$C A, C M$ & $(-0.1 / 0.2 / 0.5),(1.3 / 1.4 / 1.5)$ & $0,-1$ \\
\hline$C M, C A$ & $(-1.5 /-1.4 /-1.3),(1.3 / 1.4 / 1.5)$ & $(-0.5 /-0.4 /-0.3),(-0.7 /-0.6 /-0.5)$ \\
\hline$C M, C M$ & \multicolumn{2}{|c}{}
\end{tabular}

Nesse caso, não se sabe se o EN está na combinação $((C A, C M), C)$ ou na combinação $((C A, C M), N C)$, pois a Eq. (9) não consegue apontar qual o maior número fuzzy entre $(-0.8 /-0.4 / 0)$ e $(-0.5 /-0.4 /-0.3)$. Da mesma forma, se a relação de ordem 
Buckley $^{1}$

Uma Abordagem para Jogos Bayesianos Fuzzy com base na Probabilidade Fuzzy de definida pela Eq. (10) fosse adotada, também não se obteria sucesso na ordenação das recompensas fuzzy.

No entanto, ao se utilizar a relação de ordem de Asmus-Dimuro, definida pelas equações (17) e (18), percebe-se que $(-0.8 /-0.4 / 0)<(-0.5 /-0.4 /-0.3)$, e assim, encontra-se o EN na combinação $((C A, C M), N C)$. Ou seja, mesmo com uma menor chance da agência ser ousada (em torno de 60\%), ainda é mais aconselhável que a empresa não contrate seus serviços.

\subsection{Discussão}

Primeiramente, fica evidente uma forte similaridade entre o jogo Bayesiano fuzzy e sua versão crisp, quando se utiliza a abordagem de Buckley para cálculo de probabilidades fuzzy. Essa opção permite que os cálculos se mantenham similares aos realizados para o jogo bayesiano crisp, apenas estendendo de valores reais para intervalos reais, de acordo com a restrição aritmética.

Percebe-se também que os cores das recompensas fuzzy do jogo Bayesiano fuzzy encontradas na Tabela 8 são exatamente os mesmos valores das recompensas correspondentes do jogo bayesiano crisp contidos na Tabela 7. Isso mostra que, ao se trabalhar com números fuzzy triangulares, o jogo bayesiano fuzzy representa uma família de jogos de acordo com as probabilidades fuzzy escolhidas e que o jogo bayesiano crisp se torna um caso particular ao se tomar $\alpha=1$ para $\bar{p}[\alpha]$ e $\bar{q}[\alpha]$, ou seja, ao se eliminar a incerteza a respeito das probabilidades a serem escolhidas.

Analisando agora os extremos dos números fuzzy encontrados na Tabela 8, percebese que quanto maior o coeficiente de $\alpha$ nas expressões contidas na Tabela 6 , mais distantes do core estão os extremos do número fuzzy produzido. Como esses coeficientes são obtidos através dos cálculos das médias das recompensas contidas nas Tabelas 2 e 3, conclui-se que quanto maiores forem os módulos das recompensas, mais incerteza (distância dos extremos até o core) resultará dos cálculos das médias fuzzy.

Ressalta-se a dependência da relação de ordem adotada para números fuzzy da solução de EN. Embora as relações de ordem definidas pelas equações (9) e (10), adotadas por Buckley em $[13,14]$, tanto na introdução à sua abordagem para probabilidades fuzzy como em aplicações, sejam intuitivas e de fácil aplicação, essas relações podem não ser adequadas para a abordagem de jogos Baysianos.

Observou-se que números fuzzy com "muita" incerteza podem não ser ordenáveis segundo a Eq. (9), por exemplo, quando para $\overline{p_{a}}[\alpha]=\left[a_{1}(\alpha), a_{2}(\alpha)\right]$ e $\overline{p_{b}}[\alpha]=\left[b_{1}(\alpha), b_{2}(\alpha)\right]$, existir um $\alpha \in[0 ; 1]$ tal que $a_{1}(\alpha)>b_{1}(\alpha)$ e $a_{2}(\alpha)<b_{2}(\alpha)$, ou seja, quando para um $\alpha \in[0 ; 1]$ tem-se que $\overline{p_{a}}[\alpha] \subset \overline{p_{b}}[\alpha]$. Quanto maior for a incerteza dos resultados dos cálcu- 

ley ${ }^{1}$

los das médias fuzzy, mais chance de se obter esse tipo de situação, o que pode impossibilitar a ocorrência de um Equilíbrio de Nash.

Observe que a relação de ordem definida pela Eq. (10) também é bastante restritiva, pois, por exemplo, não ordena números fuzzy tal que existe um $\alpha \in[0 ; 1] \operatorname{com} a_{1}(\alpha)<b_{1}(\alpha)$ e $a_{2}(\alpha)<b_{2}(\alpha)$ ou $b_{1}(\alpha)<a_{1}(\alpha)$ e $b_{2}(\alpha)<a_{2}(\alpha)$.

Para contornar esse problema, pode-se escolher outro método de ordenação ${ }^{8}$. Por exemplo, a relação de Asmus-Dimuro [26], definida pelas equações (17) e (18), que por se tratar de uma relação de ordem total, sempre possibilitará uma (ou mais) soluções de EN. A escolha de qual método de ordenação a ser utilizado vai depender do problema que se está modelando.

\section{Conclusão}

A abordagem para probabilidade fuzzy de Buckley [13, 14], que toma como base a definição de uma restrição aritmética, se mostrou adequada a ser utilizada em jogos Bayesianos, produzindo assim a abordagem de jogos Bayesianos fuzzy introduzida neste artigo.

Assim, essa nova abordagem fuzzy determina diferentes famílias de jogos crisp, para diferentes probabilidades fuzzy aplicadas. Essa gama de opções pode representar um auxílio para a tomada de decisão dos agentes interessados, principalmente quando se está lidando com valores muito grandes, onde qualquer diferença de resultados, por menor que seja, é considerável.

Além disso, quando o Equilíbrio de Nash se mantém para essa família de jogos, significa que a mesma combinação de estratégias pode ser escolhida para valores próximos aos cores das probabilidades fuzzy escolhidas, ou como foi modelado neste trabalho, "em torno" desses cores.

A determinação de qual combinação de estratégias configura um EN depende fortemente de qual método de ordenação de números fuzzy se utiliza. Este artigo analisou um exemplo que duas relações de ordem adotadas por Buckley não levam a uma solução de EN.

Os números fuzzy triangulares simétricos, utilizados neste trabalho para representar probabilidades fuzzy, são de fácil manipulação e permitem a utilização da relação de ordem de Asmus-Dimuro (equações (17) e (18)) como método de ordenação, que se mostrou eficaz justamente por se tratar de uma relação de ordem total, e que privilegia o nível de incerteza das informações que se está comparando.

Assim, acredita-se que este trabalho possa contribuir para o entrelaçamento ainda

${ }_{8}^{8}$ Para um uma análise comparativa de diversos métodos de ordenação de números fuzzy, consulte [10]. 
Uma Abordagem para Jogos Bayesianos Fuzzy com base na Probabilidade Fuzzy de

maior entre a teoria dos jogos e a teoria dos conjuntos fuzzy, bem como auxiliar nas mais diversas aplicações envolvendo jogos Bayesianos.

Como trabalho futuro, pretende-se estudar jogos Bayesianos fuzzy intervalares, adotando a abordagem para probabilidade fuzzy intervalar introduzida em [47], com aplicação no desenho de leilões.

Agradecimentos. Este trabalho recebeu apoio financeiro do CNPq (Proc. 560118/10-4, 305131/20109, 476234/2011-5) e da FAPERGS (Proc. 11/0872-3). T.C. Asmus é bolsista REUNI/CAPES/FURG. Os autores agradecem aos revisores anônimos por suas sugestões e comentários.

\section{Referências}

[1] W. Amaral and F. Gomide. An algorithm to solve two-person non-zero sum fuzzy games. In O. Castillo, P. Melin, O. Montiel Ross, R. Sepúlveda Cruz, W. Pedrycz, and J. Kacprzyk, editors, Theoretical Advances and Applications of Fuzzy Logic and Soft Computing, Advances in Soft Computing, pages 296-302. Springer, Berlin, 2007.

[2] W. Amaral and F. Gomide. A coevolutionary approach to solve fuzzy games. In R. Bello, R. Falcón, W. Pedrycz, and J. Kacprzyk, editors, Granular Computing: At the Junction of Rough Sets and Fuzzy Sets, pages 121 - 130. Springer, Berlin, 2008.

[3] W. M. Amaral. Teoria de jogos nebulosos na resolução de problemas de decisão e conflito de interesses. Master's thesis, UNICAMP, Campinas, 2007.

[4] R. Aumann. Correlated equilibrium as an expression of bayesian rationality. Econometrica, 55:1-18, 1978.

[5] R. C. Barros, L. C. e Bassanezi. Tópicos de Lógica Fuzzy e Biomatemática. UNICAMP/IMECC, Campinas, 2010.

[6] B. C. Bedregal, G. P. Dimuro, R. H. N. Santiago, and R. H. S. Reiser. On interval fuzzy S-implications. Information Sciences, 180(8):1373-1389, 2010.

[7] J. Berg. Statistical mechanics of random two-player games. Physics Review E, 61:23272339, 2000.

[8] A. Blau. Random-payoff two-person zero-sum games. Operations Research, 22:12431251, 1974.

[9] G. Bojadziev and M. Bojadziev. Fuzzy Sets, Fuzzy Logic, Applications. World Scientific, Singapore, 1995. 
Uma Abordagem para Jogos Bayesianos Fuzzy com base na Probabilidade Fuzzy de Buckley ${ }^{1}$

[10] G. Bortolan and R. Degani. A review of some methods for ranking fuzzy subsets. Fuzzy Sets and Systems, 15(1):1 - 19, 1985.

[11] J. J. Buckley. Fuzzy hierarchical analysis. Fuzzy Sets and Systems, 17(3):233 - 247, 1985.

[12] J. J. Buckley. Ranking alternatives using fuzzy numbers. Fuzzy Sets and Systems, 15(1):21 - 31, 1985.

[13] J. J. Buckley. Fuzzy Probabilities: New Approach and Applications. Springer, Berlin, 2005.

[14] J. J. Buckley and E. Eslami. Uncertain probabilities I: the discrete case. Soft Computing - A Fusion of Foundations, Methodologies and Applications, 7:500-505, 2003.

[15] J. J. Buckley and L. Jowers. Fuzzy two-person zero-sum games. In Monte Carlo Methods in Fuzzy Optimization, volume 222 of Studies in Fuzziness and Soft Computing, pages 165-173. Springer, Berlin, 2008.

[16] D. Butnariu. Fuzzy games: A description of the concept. Fuzzy Sets and Systems, 1(3): $181-192,1978$.

[17] D. Butnariu. Solution concepts for n-person fuzzy games. In M. Gupta, R. K. Ragde, and R. R. Yager, editors, Advances in Fuzzy Set Theory and Applications. Kluwer, Boston, 1979.

[18] L. Campos. Fuzzy linear programming models to solve fuzzy matrix games. Fuzzy Sets and Systems, 32:275-289, 1989.

[19] G. Cassidy, A. Field, and L. Kirby. Solution of a satisfying model for random payoff games. Management Science, 19:266-271, 1972.

[20] P. T. Chang and E. S. Lee. Fuzzy arithmetic and comparison of fuzzy numbers. In M. Delgado, J. Kacprzyk, J. L. Verdegay, and M. A. Vila, editors, Fuzzy Optimization: Recent Advances, pages 69-81. Physica-Verlag, Heidelberg, 1994.

[21] A. Charnes, M. Kirbyand, and W. Raike. Zero-zero chance-constrained games. Theory of Probability and its Applications, 13:628-646, 1968.

[22] G. Chen and T. T. Pham. Introduction to Fuzzy Sets, Fuzzy Logic, and Fuzzy Control Systems. CRC Press, New York, 2000.

[23] J.-F. Dang and I-H. Hong. The cournot game under a fuzzy decision environment. Computers and Mathematics with Applications, 59(9):3099-3109, 2010. 


\section{Buckley $^{1}$}

Uma Abordagem para Jogos Bayesianos Fuzzy com base na Probabilidade Fuzzy de

[24] G. P. Dimuro, B. C. Bedregal, R. H. N. Santiago, and R. H. S. Reiser. Interval additive generators of interval t-norms and interval t-conorms. Information Sciences, 181(18):3898 - 3916, 2011.

[25] G. P. Dimuro, A. C. R. Costa, , and D. M. Claudio. A coherence space of rational intervals for a construction of IR. Reliable Computing, 6(2):139-178, 2000.

[26] T. C. Asmus e G. P. Dimuro. Uma ordem total para números fuzzy triangulares simétricos. In Anais do CNMAC 2012, Águas de Lindóia, 2012, São Carlos, 2012. SBMAC. (artigo aceito, a ser publicado).

[27] R. Fiani. Teoria dos Jogos: para cursos de administração e economia. ElsevierCampus, Rio de Janeiro, 2010.

[28] J. Gao. Credibilistic game with fuzzy information. Journal of Uncertain Systems, 1(1):74 - 80, 2007.

[29] J. Gao, Z.-Q. Liu, and Y.-K. Liu. Equilibrium strategies of two-player nonzero-sum games with fuzzy payoffs. In Proceddings of the IEEE International Fuzzy Systems Conference, FUZZ-IEEE 2007, pages 1 -5, Los Alamitos, 2007. IEEE.

[30] D. Garagic and J. B. Cruz. An approach to fuzzy noncooperative nash games. Journal of Optimization Theory and Applications, 118:475-491, 2003.

[31] P. Grzegorzewski. Metrics and orders in space of fuzzy numbers. Fuzzy Sets and Systems, 97(1):83 - 94, 1998.

[32] C. Harsanyi. Games with incomplete information. The American Economic Review, 85:291-303, 1999.

[33] F. Kacher and M. Larbani. Existence of equilibrium solution for a non-cooperative game with fuzzy goals and parameters. Fuzzy Sets and Systems, 159(2):164 - 176, 2008.

[34] M. Larbani. Non cooperative fuzzy games in normal form: A survey. Fuzzy Sets and Systems, 160(22):3184 - 3210, 2009.

[35] K. Leyton-Brown and Y. Shoham. Essentials of game theory: A concise, multidisciplinary introduction. Morgan \& Claypool, http://www.morganclaypool.com/, 2008.

[36] S.-T. Liu and C. Kao. Solution of fuzzy matrix games: An application of the extension principle. International Journal of Intelligent Systems, 22(8):891-903, 2007.

[37] R. D. Luce and H Raiffa. Games and Decisions: Introduction and Critical Survey. Dover, New York, 1989. 
Uma Abordagem para Jogos Bayesianos Fuzzy com base na Probabilidade Fuzzy de Buckley ${ }^{1}$

[38] T. Maeda. Characterization of the equilibrium strategy of the bimatrix game with fuzzy payoff. Journal of Mathematical Analysis and Applications, 251(2):885 - 896, 2000.

[39] T. Maeda. On characterization of equilibrium strategy of two-person zero-sum games with fuzzy payoffs. Fuzzy Sets and Systems, 139(2):283 - 296, 2003.

[40] R. E. Moore. Methods and Applications of Interval Analysis. SIAM, Philadelphia, 1979.

[41] R. E. Moore, R. B. Kearfott, , and M. J. Cloud. Introduction to Interval Analysis. SIAM, Philadelphia, 2009.

[42] R. B. Myerson. Refinements of the Nash equilibrium concept. International Journal of Game Theory, 7:73-80, 1978.

[43] J. Nash. Equilibrium points in n-person games. Proceedings of the National Academy of Sciences, 36(1):48 - 49, 1950.

[44] A. M. Nejad and M. Mashinchi. Ranking fuzzy numbers based on the areas on the left and the right sides of fuzzy number. Computers \& Mathematics with Applications, 61(2):431 - 442, 2011.

[45] H. T. Nguyen and E. A. Walker. A First Course in Fuzzy Logic. Chapman \& Hall/Crc, Las Cruces, third edition, 2006.

[46] I. Nishizaki and M. Sakawa. Fuzzy and Multiobjective Games for Conflict Resolution. Physica-Verleg, Heidelberg, 2001.

[47] J. L. B. Ribes, T. C. Asmus, and G. P. Dimuro. Sobre probabilidades fuzzy intervalares. In Anais do CNMAC 2012, Águas de Lindóia, 2012, São Carlos, 2012. SBMAC. (artigo aceito, a ser publicado).

[48] D. Roberts. Nash equilibria of cauchy-random zero-sum and coordination matrix games. International Journal of Game Theory, 34:167-184, 2006.

[49] T. J. Ross. Fuzzy Logic with Engineering Applications. Wiley, New Mexico, 2004.

[50] Y. Shoham and K. Leyton-Brown. Multiagent Systems: Algorithmic, Game-Theoretic, and Logical Foundations. Cambridge University Press, Cambridge, 2009.

[51] V. Vijay, S. Chandra, and C.R. Bector. Matrix games with fuzzy goals and fuzzy payoffs. Omega, 33(5):425 - 429, 2005.

[52] J. von Neumann and O. Morgenstern. Theory of Games and Economic Behavior. Wiley, New York, 1944. 
Uma Abordagem para Jogos Bayesianos Fuzzy com base na Probabilidade Fuzzy de

[53] . Wang and E. E. Kerre. Reasonable properties for the ordering of fuzzy quantities (I). Fuzzy Sets and Systems, 118(3):375 - 385, 2001.

[54] C. Wang, W. Tang, and R. Zhao. Static bayesian games with finite fuzzy types and the existence of equilibrium. Information Sciences, 178(24):4688 - 4698, 2008.

[55] X. Wang and E. E. Kerre. Reasonable properties for the ordering of fuzzy quantities (II). Fuzzy Sets and Systems, 118(3):387 - 405, 2001.

[56] M. Wooldridge. An Introduction to Multiagent Systems. Wiley, West Sussex, 2009.

[57] H. Wu and M. Parlar. Games with incomplete information: A simplified exposition with inventory management applications. International Journal of Production Economics, 133(2):562 - 577, 2011.

[58] L. Xu, R. Zhao, and Y. Ning. Two-person zero-sum matrix game with fuzzy random payoffs. In De-Shuang Huang, Kang Li, and George Irwin, editors, Computational Intelligence, volume 4114 of Lecture Notes in Computer Science, pages 809-818. Springer Berlin / Heidelberg, 2006.

[59] L. Xu, R. Zhao, and T. Shu. Three equilibrium strategies for two-person zero-sum game with fuzzy payoffs. In Lipo Wang and Yaochu Jin, editors, Fuzzy Systems and Knowledge Discovery, volume 3613 of Lecture Notes in Computer Science, pages 478478. Springer, 2005.

[60] L. A. Zadeh. Fuzzy sets. Information and Control, 8(3):338-353, 1965.

[61] L. A. Zadeh. The concept of a linguistic variable and its application to approximate reasoning - I. Information Sciences, 8(3):199-249, 1975. 